\title{
EVOLUCIÓN DE LA DEMANDA POR DINERO EN CHILE (1974-86): UNA APLICACIÓN DEL FILTRO DE KALMAN*
}

\author{
Raul Labán**
}

\section{Sumario}

El propósito de este artículo es estudiar la evolución de la demanda por dinero en Chile, durante el periodo (1974-1986), el cual está lleno de reformas estructurales y cambios políticos.

Este articulo intenta presentar una evidencia empírica acerca de la estabilidad y predictibilidad de esta demanda, por un país expuesto a instabilidades continuas. La aplicación del Filtro de Kalman sirve a nuestro propósito de estimación de elasticidades váriables.

\section{Abstract}

The purpose of this paper is to study the evolution of the demand for money in Chile during a period (1974-1986) which is plenty of structural reforms and policy changes.

This paper attempts to present empirical evidence with respect to the stability and predictability of this demand for a country exposed to continuous instabilities. The application of the Kalman Filter serves our purpose of estimating changing elaticities.

\section{Introducción}

El objetivo central de este estudio es analizar la evolución de la demanda por dinero, en un periodo caracterizado por profundas trans-

- Este trabajo forma parte del área de investigaciones de CIEPL AN sobre Macroeconomfa y Empleo, y ha tenido el apoyo del Centro Internacional de Investigaciones para el Desarrollo (CIID-IDRC). Una versión preliminar de este trabajo fue presentada en el VII Congreso Latinoamericano de la Sociedad Econométrica, São Paulo, Brasil, agosto 4-7, 1987. Se agradecen los comentarios recibidos alli, y muy en particular los de Pedro Valls, Klaus Schmidt-Hebbel, Roberto Zahler, Manuel Marfán y Andrés Velasco. El autor desea agradecer, en forma muy especial, a Patricio Meller, quien guió el desarrollo de mi tesis, de la cual el presente es un resúmen, y a Cornelio González, por la ayuda brindada en su revisión. El único responsable por su contenido es el autor.

* "Corporación de Investigaciones Economica para Latinoamerica - CIEPLAN.

R. de Econometria Rio de Janeiro v. VIII, n. 1, p. $45-82$ jan./jun. 1988 
formaciones institucionales, sociales y ec.onómicas, como las vividas por Chile a partir de 1974.

La estabilidad y predictibilidad de dicha demanda juegan un rol predominante en la formulación, implementación y evaluación de la efectividad de la política monetaria. Lamentablemente, existe poca evidencia empírica para economías en desarrollo en que el contexto macroeconómico, en términos generales y las reglas de política económica están insertas en un contínuo proceso de cambio. Adicionalmente, los pocos estudios con que se cuenta parten invariablemente del supuesto a priori de coeficientes fijos. Esto no nos permite, como se demuestra más adelante, capturar la naturaleza cambiante de nuestras economfas. Más aún, las técnicas estáticas y tradicionales de estimación, basadas en este supuesto, como son los Mínimos Cuadrados Ordinarios ( MCO ), pueden proporcionar resultados cuya interpretación económica y numérica resulte ser totalmente errónea, al igual que las predicciones y evaluaciones que de ellas se deriven.

Con este trabajo se pretende contribuir a superar este vacío. Para ello aplicamos al caso chileno la técnica dinámica de estimación del Filtro de Kalman (F\&), la cual nos permite relajar este supuesto a priori de constancia en los parámetros. Los resultados que de esta aplicación se obtienen son comparados con aquellos que se obtendrían de haberse usado somo técnica de estimación e inferencia los MCO.

\section{Marco Analitico-Teorico}

La eșabilidad y predictibilidad de la demanda por dinero juega un rol cesstral en la formulación, implementación y evaluación de la polf́tica monetaria. Esto no significa que la demanda por dinero sea constante, sino que la cantidad deseada de dinero responde, en forma predecible, ante cambios en las variables explicativas.

Los estudios econométricos tradicionales de estimación y de análisis de estabilidad parten del supuesto a priori de constancia en los parámetros, que describen las relaciones estructurales básicas entre distintas variables económicas. Estos métodos de estimación permiten, a lo sumo, obtener un único estimador, constante a lo largo de todo el período muestral, para cada coeficiente.

Una evidencia cada vez mayor indica que el supuesto tradicional de regresión, de constancia en los parámetros, no es generalmente válido. Cooley y Prescott (1973) afirman que sostener relaciones tecnológicas y de comportamiento estables en el tiempo, en muchos casos, no sólo resulta ser una premisa heroıca sino, también, completamente inconsistente con la teoría económica.

Estas fluctuaciones a que están sometidos los sistemas económicos son aún más acentuadas en países en desarrollo, que enfrentan 
cambios contínuos y profundos en el contexto macroeconómico, instirucional y político.

Siguiendo lá línea de argumentación planteada por Lucas (1970), pero introduciendo las modificaciones necesarias para considerar otros aspectos, además de la influencia de la política económica sobre la estructura de las relaciones econométricas, se podria inferir que, en una economía expuesta a transformaciones y reformas de la magnitud de las vividas por Chile en el período post-1973, deberían esperarse grandes inestabilidades en los coeficientes de las ecuaciones que relacionan causalmente a distintas variables económicas ${ }^{1}$.

Esto significa que las estimaciones econométricas tradicionales, que descansan sobre el supuesto a priori de constancia en los parámetros y que no consideran estos cambios en el contexto macroeconómico, pueden proporcionar resultados cuya interpretación económica y numérica resulte ser totalmente errónea, al igual que las predicciones y evaluaciones que de ellos se deriven.

El período 1973-86 se ha caracterizado por profundas transformaciones y reformas en la economía chilena, las cuales tuvieron (y siguen teniendo) importantes repercusiones sobre el mercado monetario doméstico.

Estas reformas y cambios de política económica, que se han concentrado en un período relativamente corto (1973-86), no permiten sostener la validez y relevancia de los resultados de los análisis econométricos aplicados al estudio del mercado monetario chileno, ya que éstos parten invariablemente suponiendo un vector de coeficientes fijo. Es decir, parten restringiendo el estudio a agentes económicos "irracionales" que, aún cuando capaces de predicir y anticiparse a la evolución de las variables de política y a los cambios que se puedan dar en el contexto macroeconómico e institucional, no adapian sus planes a esta nueva información.

El modelo de demanda por dinero utilizado corresponde a la función convencional de demanda por dinero de largo plazo del tipo:

$$
M_{t}=L\left(Y_{t}^{*}, C_{t}^{*}\right)
$$

en donde, $M_{t_{*}}$ representa el stosk deseado de saldos reales de largo plazo en $t, \boldsymbol{Y}_{\boldsymbol{t}}^{*}$ el producto doméstico esperado expresado en precios constantes en \&, y $C_{\text {\& }}^{*}$ el costo esperado de mantener dinero en el período $\mathbf{t}$.

${ }^{1}$ Según Lucas, hay evidencia empírica y teórica suficiente para sostener que una estructura que asume coeficientes fijos no debe utilizar-se con fines predictivos, ni de evalueción de la efectividad de la política económica. 
Siguiendo a Cagan (1956), se adopta la siguiente forma funcional, en términos logarítmicos, para la ecuación $(1)^{2}$,

$$
\operatorname{Ln} M_{t}=\operatorname{Ln} A+\beta_{1} \operatorname{Ln} Y_{t}^{*}+\beta_{2} C_{t}^{*}
$$

La interpretación económica de la expresión ( 2 ) indicaria que existen dos elementos centrales que afectan la demanda de largo plazo por saldos reales: ( $\mathrm{i}$ ) el ingreso esperado y ( ii ) el costo esperado de mantener dinero, que influye negativamente sobre el ş́ck deseado de dinero ${ }^{3}$.

Se estima la ecuación ( 2 ) a través de la técnica tradicional estática de mínimos cuadrados ordinarios ( MCO ) y a través del algoritmo del Filtro de Kalman ( FK), el cual permite la estimación econométrica de "parámetros variables".

Dado lo poco utilizado que ha sido el FK, procedemos a desarrollar brevemente sus principales elementos metodológicos ${ }^{4}$.

\section{Elementos Matodológicos dal Filtro de Kalman}

\section{1 - Descripción dal problema}

La característica central de este método es que el vector de parámetros $\beta_{t}$, que es potencialmente distinto para cada periodo $t$, está vinculado al vector de parámetros de periodos anteriores (a través de una matriz de transición que se supone conocida a priori) y a elementos de innovación que permiten capturar la historia estocástica de la economia.

Suponemos que se tiene un sat de variables estado ("estado de la naturaleza") que cambian a través del tiempo, que no son directamente observables y están sujetas a una distorción sistemática así como a una contaminación por "ruído". Por otro lado, sea $Y_{\hat{t}}$ una variable que sl se observa y que está relacionada a las variables de estado a través de una "ecuación de medición". Si las variables de estado están contenidas en un vector $m \times 1, \beta_{\Re}$, esta ecuación puede escribirse como,

${ }^{2}$ Suponiendo coeficientes constantes, esta estructura impone como restricción a priori una elasticidad constante con respecto a $Y_{q}^{a}$ y una elasticidad creciente con respecto a $C_{t}^{\prime \prime}$ (Cagan, 1956).

${ }^{3}$ Ocasionalmente, se permite la existencia do desequilibrios de stocks en el corto plazo, es decir, que el ajuste del stock deseado en efectivo no es instantáneo (ver Corbo, 1974, 1981, Matte y Rojas, 1986 y Roley 1985). En este trabajo, son los coeficientes los que capturan estos desequilibrios como cualquier otro ajuste frente a un cambio en las variables exógenas.

${ }^{4}$ Un anślisis más detällado se puede encontrar en Harvey (1981), Chows (1983), Sarris (1973) y Labán (1987a). 


$$
Y_{t}=x_{t} \beta_{t}+U_{t} \quad U_{t} \sim N\left(0, \sigma_{\cdot d t}^{2}\right) \quad \nabla_{t}
$$

donde $X_{t}$ es un vector fijo $1 \times \mathbf{m}$.

El vector de estado, $\beta_{t}$, no es directarnente observable, pero se supone que sus variaciones están gobernadas por un proceso bien definido, representado por la "ecuación de transición", que corresponde a una estructura markoviana de primer orden ${ }^{5}$.

$$
\beta_{\mathrm{t}}=T \beta_{\mathrm{t}-1}+\mathrm{V}_{\mathrm{t}} \quad \mathrm{V}_{\mathrm{t}} \sim \mathrm{N}\left(\mathrm{O}, \sigma_{\mathrm{vt}}^{2}\right) \quad \nabla_{\mathrm{t}}
$$

donde $T$ es una matriz fija $m \times m^{6}$.

Adicionalmente, se supone que las perturbaciones de ambas ecuaciones no están serial ni contemporáneamente correlacionadas, ni tampoco con el vector de estado inicial, $\beta_{0}{ }^{7}$.

El hecho de existir un $\beta_{\hat{\imath}}$ potencialmente distinto para cada período $\uparrow$ imposibilita su estimación a través de la técnica traaicional de $\mathbf{M C O}^{8}$. Es decir, no hay forma de estimar el vector $\beta_{\hat{\mathbf{t}}}$, a menos que se disponga de una mayor información del proceso generador del vector de estado, el cual se refleja en la estructura de la ecuación de transición.

Una de las posibles formas que puede tomar esta ecuación es la supuesta en $(4)$; sin restringir los coeficientes a una determinada estructura es imposible su estimación ${ }^{9}$. La selección de dicha estructura se debe a que permite, de una forma relativamente sencilla, combinar dos elementos centrales: ( $i$ ) la in fluencia de la estructura pasada y ( ii ) la incidencia de los elementos estocásticos - ya que posibilita un amplio rango de simulaciones sobre ellos. Incluso, cabe señalar que la combinación de una matriz de transición $T$ igual a la identidad, con un vector $\boldsymbol{V}_{\hat{\mathbf{t}}}$ nulo, reduce la técnica de estimación del $F K$ al método tradicional MCO. En consecuencia, el supuesto a priori de constancia de los parâmetros de un modelo de regresión es simplesmente un caso particular del método del FK.

${ }^{5}$ Una especificación más general se encuentra en Harvey (1981).

${ }^{6} \mathrm{~A}$ nivel téorico, esta matriz también puede ser variable $\left(\gamma_{\mathrm{q}}\right)$. Aún cuando sea posible la resolución de este problema en tales circunstancias, es imposible su estimeción sin la imposición de restricciones adicionales a su distribución dinámica. Ver Harvey (1981) y Sarris (1973).

${ }^{7}$ El desarrollo para el caso multi-ecuacional se encuentra en Labán (1987a).

${ }^{8}$ Una demonstración formal se encuentra en Sarris (1973).

Distintas estructuras de la ecuación de transición se pueden encontrar en Harvey (1981), Sarris (1973) y Engle y Watson (1985). 


\section{2 - Estimación de las restricciones "a priori"}

Con la estructura adoptada en $(4)$, el vector de estado $\left(\beta_{4}\right)$ está restringido a un patrón particular de evolución en el tiempo. Sin embargo, se ignora el nivel del cual parte este patrón y la dispersión que puede tener en la base $y$, por lo tanto, a través del tiempo. Así, el problema se compone de dos etapas: $(i)$ encontrar un $\beta$ inicial, $\left(\beta_{0}\right)$ que mejor se ajuste a los datos de la base, y la matriz de covarianza asociada a este, $\left(P_{0}\right)$. Es decir, obtener la distribuición "a priori" del vector $\beta_{t} ;$ ( ii ) luego se calcula la distribuición "a poswriori" del vector de estado $\beta_{\hat{q}}$. ocupando esta información a través del algoritmo del FK.

Para poder aplicar el $F K$ se requiere conocer la matriz $T, \beta_{0}$. $\sigma_{\text {wo }}^{2}$ y $\sigma_{\text {wo. }}^{2}$. Con excepción de la matriz $T$, las otras restricciones a Grieri se obtienen directamente de los datos. El mecanismo utilizado para obtener los valores iniciales para $\beta_{0}$ y $\theta_{0}\left(0_{v 0}^{2} / \sigma_{\mathrm{wO}}^{2}\right)$ es desarrollado detalladamente en Sarris (1973). Sin embargo, es necesario resaltar que los residuos de estas estimaciones no están bien comportados, presentando problemas de aurocorrelación y de heteroscedasticidad. Con el fir de obrener estimadores MAELI ${ }^{10}$, se sugiere utilizar mínimos cuadrados generalizados (AMCG). Si, además, se cumple que $U_{\hat{\vartheta}} \quad \boldsymbol{V} \quad \boldsymbol{V}_{\hat{\tau}}$ se distribuyen normalmente, el estimador de $\beta_{0}$ será igual al obtenido por el método de máxima verosimilitud ( MMV ) y tendrá la propiedad de ser MVVE ${ }^{11}$. El estimador de $\sigma_{\text {uo }}^{2}$ obtenido por MACG es un estimador insesgado; no así el obtenido por. $\mathrm{MgV}$, aunque el sesgo tiende a desaparecer para un $T$ grande.

De estit forma, se han obtenido los estimadores de $\beta_{0}$ y $\sigma_{\text {uo }}^{2}$, pero éstos esłán condicionados al valor de $\theta$. Por esta razón, es fundamental examinar si el valor encontrado para estas variables depende o no del valor de $\theta$ seleccionado. Con este fin, se ha llevado a cabo un análisis de sensibilidad con respecto al valor de este parámetro.

Solamente queda por establecer una estructura a priori para la matriz de transición $T$, para iniciar el análisis de estimación de los coeficientes variables. Dado que no existe un mecanismo convincente para seleccionar esta matriz se asume una cierta estructura, y luego se realiza un análisis de sensibilidad com respecto a ella, con el fin de comprobar la dependencia o no de los resultados encontrados a la estructura seleccionada.

\footnotetext{
10 MELI: mejor estimador lineal insesgado ( BLUE ).

11 mavB: estimador de mínima varianza entre los estimadoras insesgados, sean o no lineares.
} 


\section{3 - Estimación de los coefícientes variables}

En esta sección se proporcionan el sistema de ecuaciones de actualización para la variable de estado y su matriz de covarianza. EI papel que juegan estas ecuaciones es combinar la nueva información en $y_{t}$ coi la información ya disponible en los predictores óptimos,

$$
\beta_{t / t-1}=T \beta_{t-1} \quad t=1, \ldots, T
$$

para el vector de estado, $y$

$$
P_{t / t-1}=T P_{t-1} T^{\prime}+Q_{t} \quad t=1, \ldots T
$$

para su matriz varianza-covarianza.

Los estimadores de $\beta_{t}$ y de su matriz de covarianza, que minimizan el error cuadrático medio lineal (ECML) ${ }^{12}$, se obtienen, recursivamente, a través del algoritmo del FK de la siguiente forma,

$$
\begin{aligned}
& \beta_{t}=\beta_{t / t-1}+P_{t / t-1} x_{t} F_{t} \\
& \quad P_{t}=P_{t / t-1}-P_{t / t-1} x_{t} F_{t}^{-1} x_{t} P_{t / t-1}
\end{aligned}
$$

donde,

$$
F_{t}=X_{t} P_{t / t-1} X_{t}+H_{t} \quad t=1, \ldots, T
$$

Las ecuaciones ( 7 ), ( 8 ) y ( 9 ) componen el sistema de ecuaciones de actualización ${ }^{13}$.

En resumen, el FK es un set de ecuaciones, que permite que los coeficientes $y$ predicciones se actualicen cuando nuevas observaciones se hacen disponibles. Este proceso se realiza en dos etapas. Primero, se forma el predictor óptimo de las observaciones siguientes, dada toda la información hasta ese momento disponible. Luego, se incorpora la nueva observación al estimar el vector de estado, usando las ecuaciones de actualización ( 7 ). ( 8 ) y (9). El FK da una solución óptima al problema de actualización y de predicción. Si las observaciones se distribuyen normalmente $y$ si los estimadores corrientes del vector de estado son los mejores disponibles, el estimador de predicción y el de actualización también lo serán. .Es decir, si la estimación inicial es MVB también lo serán las obtenidos en las ecuaciones ( 5 ) y ( 7 ). En ausencia del supuesto de normalidad se da un resultado similar, pero

12 La derivación y las propiedades de estos estimadores se encuentran en Harvey (1981), Chow (1983) y en Watson (1983).

${ }^{13} \mathrm{El}$ desarrollo formal de este sistema se encuentra en Harvey (1981). 
sólo en la clase de estimadores y predictores lineales insesgados (MELI) $)^{14}$

\section{Resultados Empiricos}

\section{1 - Anălisis descrip tivo del periodo (1974.1 - 1986.4)}

El método dinámico del $\mathbf{F K}$, al igual que el método tradicional de MCO, ha sido aplicado para la estimación de la demanda por dinero en la economía chilena. Se han utilizado observaciones trimestrales para el período comprendido entre 1974 y 1986. La forma reducida básica estimada es la ecuación ( 2 ), anteriormente derivada.

Para representar los saldos reales deseados $\left(\mathbf{M}_{\mathbf{t}}\right)$, se utilizó la definición tradicional de dinero privado $(\mathbf{M} 1)^{15}$. Por otra parte, se utilizó la serie de PGB trimestral, medido en millones de pesos de $1977^{16}$, como una aproximación del ingreso esperado. Por su parte, el costo esperado de mantener dinero fue representado a través de la tasa de interés nominal de captación de 30 a 89 días no reajustables.

\section{a) Estimación de las restricciones "a priori"}

Para la obtención de los estimadores a priori que se requieren para la aplicación del método del FK, se ha efectuado lo siguiente: (i) La estimación de MCG aplicado a toda la muestra 1974-86, proporciona los estimadores iniciales para el vector $\beta_{0}$ y para $\sigma_{\text {uo }}^{2}$ para la ecuación ( 2 ). Como se señalara en la sección anterior, los estimadores obtenidos para la ecuación ( 2 ) son insesgados, consistentes y de varianza mínima. ( ii ) Para el parámetro $\theta$ se ha utilizado un valor base de 0,2 , bajo el supuesto de que los agentes económicos no reaccionan de una forma tan aleatoria ante cambios de política económica. Posteriormente, se han realizado análisis de sensibilidad para valores alternativos de $\theta$, cuyo rango fluctúa entre 0,05 y 0,7 . Valores pequeños de $\theta$ (por ej. 0,05 ) corresponderían al caso cercano a la constancia de los parámetros (agentes económicos con planes fijos). mientras que valores grandes de $\theta$ (por ej. 0,7) corresponderían a reacciones (casi) instantáneas a cambios en las reglas y anuncios de la política económica, o a la evolución proyectada del contexto macro.

\footnotetext{
${ }^{14}$ Dada la estocasticidad del vector de estado, es necesario tener cuidado con la interpretación del resultado obtenido. Para un análisis acabado de estas propriedades de los estimadores, ver Harvey (1981).

15 Para 1974-85, Vial y Marín (1986), para el año 1986 la serie se obtuvo empalmando por la tasa de variación oficial, presentada por el Banco Central de Chiie.

${ }^{16}$ Para 1974-85 los datos del PGB provienen de Arrau (1986), para el año 19\&ะ la serie se obtuvo empalmando por la tasa de variación oficial de dicha serie.
} 
económico e institucional freglas de decisiones adaptativas o racionales). En la medida en que las reglas de política se van haciendo menos predecibles, por parte de los agentes económicos, y hay un mayor grado de incertidumbre con respecto a la futura evolución de las principales variables macroeconómicas, la inestabilidad de los parámetros tiende a aumentar (mayor "ruido" en la ecuación de transición). ( iii ) Con respecto a la matriz de transición $T$, se ha usado una matriz diagonal (implícitamente se supone independencia entre los coeficientes), con el valor de 0,8 como elemento constante. Posteriormente, se han realizado análisis de sensibilidad para los valores de la diagonal de $T$, que fluctúan entre 0,5 y 0,9 . Una matriz $T$ diagonal, que sea igual a la matriz identidad, implica que cualquier cambio que experimenta el vector de coeficientes $\beta_{t}$ con respecto al vector $\beta_{t-1}$ se debe exclusivamente a factores aleatorios, i.e., el vector de coeficientes sigue una trayectoria del tipó random walk. Si conjuntamente a una matriz $T$ igual a la identidad la matriz $O_{t}$ es una matriz nula, el método del FK se reduce al procedimiento econométrico tradicional de MCO con una aplicación recursiva. Por otra parte, valores de los elementos de la diagonal, que sean my pequeños, (i.e., inferiores a $0,5)$, le asignarán una pequeña incidencia a los valores trimestrales de períodos anteriores; la estructura del pasado pierde rápidamente su influencia en el comportamiento actual.

\section{b) Estimación de los coeficientes variables}

Los resultados econométricos obtenidos se resumen en los gráfi$\cos 1$ a 4. En éstos se observa la evolución de los coeficien tes variables estimados por el FK, el estimador único MCO (bajo la hipótesis nula de parámetros constantes) y los límites superiores e inferiores (calculados con un intervalo de confianza de un $90 \%$, basado en el error estándar estimado y los valores críticos del estadígrafo $-t$ ). Esto nos permite medir la significancia estadística de la variabilidad de los coeficientes. Si la estimación por el FK en cualquier período $t$ está fuera del intervalo de confianza, la variación será significativamente distinta a la que uno toleraría bajo el supuesto de coeficientes constantes $^{17}$.

De estos resultados se puede desprender, en primera instancia y en líneas generales, que $(i)$ los. valores obtenidos de las elasticidades muestran variaciones y fluctuaciones aparentemente importantes; ( ii ) el estimador único, obtenido por el procedimiento MCO, no ilustra ni sintetiza de manera satisfactoria las variaciones que experimentan

17 Para una explicación formal de este test de estabilidad, ver Corbo y McNelis (1987) y Labán (1987a). 
las elasticidades ${ }^{18} ;(\mathrm{iii})$ las proyecciones de los deseos de liquidez. basadas en estos estimadores únicos, con fines de diseño de la política monetaria, pueden ll evar a resultados erróneos; y ( iv ) las evaluaciones de la efectividad de la política monetária ${ }^{19}$ debieran considerar este comportamiento cambiante de los agentes económicos, los cuales van ajustando sus decisiones y planes frente a cambios en las reglas de política y en el contexto prevaleciente, lo que depende estrechamente de la información de que se disponga y de como sean visualizados estos shocks.

En relación a la evolución de los coeficientes de la demanda por saldos reales, se observa una gran sensibilidad de ellos a los cambios ocurridos en el contexto macroeconómico e institucional y a los cambios en las reglas de la política económica. Tanto la constante como la elasticidad ingreso de esta función de demanda capturan fuerte inestabilidad durante el año 1981. Por otra parte, la semi-elasticidad interés captura inestabilidades "significativas" durante los años 1974-76 y a partir del año 1983 hasta la fecha. Por último, la elasticidad costo muestra variaciones significativas entre 1974-77, 1982.2, 1983.2 y a partir de 1986.

Es decir, además del período 1977-80, uno o mäs de los coeficiente $5^{20}$ de la función de demanda por dinero muestran variaciones "significativas" con respecto a los intervalos permitidos por el supuesto de constancia (a priori) de los coeficientes. Esto lleva a rechazar la hipótesis nula de estabilidad de la función de demanda por dinero entre 1974-86, para Chile, siendo el período 1977-80 un periodo "relativamente" más estable.

Por otro lado, cabe mencionar que los errores estándar de la regresión por MCO de la constante y de la elasticidad ingreso son muy al tos, un $179 \%$ y $20 \%$ respectivamente, lo cual nos lleva a cuestionar el hecho de que coeficientes que puedan variar entre estos intervalos (la constante entre $-2,89$ y $-7,89$ y la elasticidad ingreso entre $1,12$ y 1,79$)$ puedan considerarse estables.

${ }^{18}$ A una conclusión similar llegan Meller y Labán (1987) al analizar el mercado laboral chileno entre el período 1974-85.

${ }^{19}$ Un marco analítico equivalente debiera utilizarse para la evaluación de otras herramientas de la política económica (Lucas, 1976).

${ }^{20}$ De la ecuación ( 2 ) se obtiene el estimador MCO único para la constante, la elasticidad ingreso y para la semi-elasticidad interés. Dado que la especificación de ésta no permite obtener una única elasticidad costo para todo el periodo muestral, se estima el seguiente modelo por MCO,

$$
\operatorname{Ln} M_{t}=\alpha_{0}+\alpha_{1} \operatorname{Ln} Y_{t}^{e}+\alpha_{2} \operatorname{Ln} C_{t}^{e}
$$

del cual se obtiene una elasticidad interés $\left(\alpha_{2}\right)$, constante para todo el perfodo. Para mayores detalles ver Labán (1987a). 
Los resultados obtenidos podrian ilustrar el impacto que han tenido, sobre la demanda por saldos reales, los distintos contextos macroeconómicos e institucionales y los contínuos cambios en las reglas de la política económica del período 1974-86. Estos habrían afectado significativamente la estructura del mercado monetario y el comportamiento de los demandantes de dinero. Se pueden distinguir los siguientes entornos: (i ) 1974-76, período recesivo de altas tasas 'de inflación y de interés, sujeto a una política macroeconómica contractiva estabilizadora anti-inflacionaria, con un mercado monetario $y$ financiero poco desarrollado y con una baja integración a los mercados internacionales de bienes y activos. Un período caracterizado por modificaciones transcendentales en los principios básicos sobre los cuales se conduciría la política económica, lo que llevó a un cambio radical en la estructura de propiedad (i. e., privatización de bancos comerciales) y una reorientación de la actividad económica. ( ii ) 1977-81, período expansivo de apertura comercial y liberalización financiera. Durante este período se observa un aumento explosivo del flujo de capitales extranjeros hacia Chile, facilitado por la creciente confianza de los agentes económicos en las reglas de la política cam- biaria y financiera, lo que llevó a una fuerte reducción del costo esperado de endeudamiento en moneda ex tranjera - y por la pradigalidad de la banca comercial extranjera para colocar sus excesos de liquidez en la región. En el mismo período la tasa de interés en pesos, aún cuando disminuyó, siguió siendo alta. En este período, se observa una importante expansión de la actividad financiera y una creciente confianza en el manejo económico de la autoridad. ( iii ) 1982-83, Chile experimenta una nueva fase recesiva, caracterizada por un gran desequilibrio en las cuentas externas. A mediados de 1981, se produce un aumento en las tasas de interés internacionales ${ }^{21}$ y una disminución en el poder adquisitivo de las exportaciones, de alrededor de un $20 \%$. La autoridad monetaria piensa que esto es un problema coyuntural de caráter transitorio, mantiene equivocadamente la política de tipo de cambio fijo y fuerza a la economía a un ajuste en su nivel de gastos vía aumentos de las tasas de interés. A mediados de 1982 se agota el crédito externo y en junio de ese año se devalúa la moneda nacional, en forma tardía e insuficiente. Los agentes económicos así lo advierten, y el Banco Central ve disminuir sus reservas internacionales en 440 millones de dólares en menos de un mes. A pesar de las sucesivas depreciaciones del peso y las crecientes restricciones cambiarias, las reservas internacionales siguen disminuyendo vertiginosamente duran-

\footnotetext{
${ }^{21}$ Con un endeudamiento externo de más de 15 mil millones de dólares, contraido en gran parte a una tasa de interés flotante, lo cual lleva no sólo a un aumento del costo del endeudamiento marginal sino también sobre el total del monto de la deuda previamente contraída a esta tasa variable.
} 
Gráfico 3

EVOLUCION DE LA "CONSTANTE"

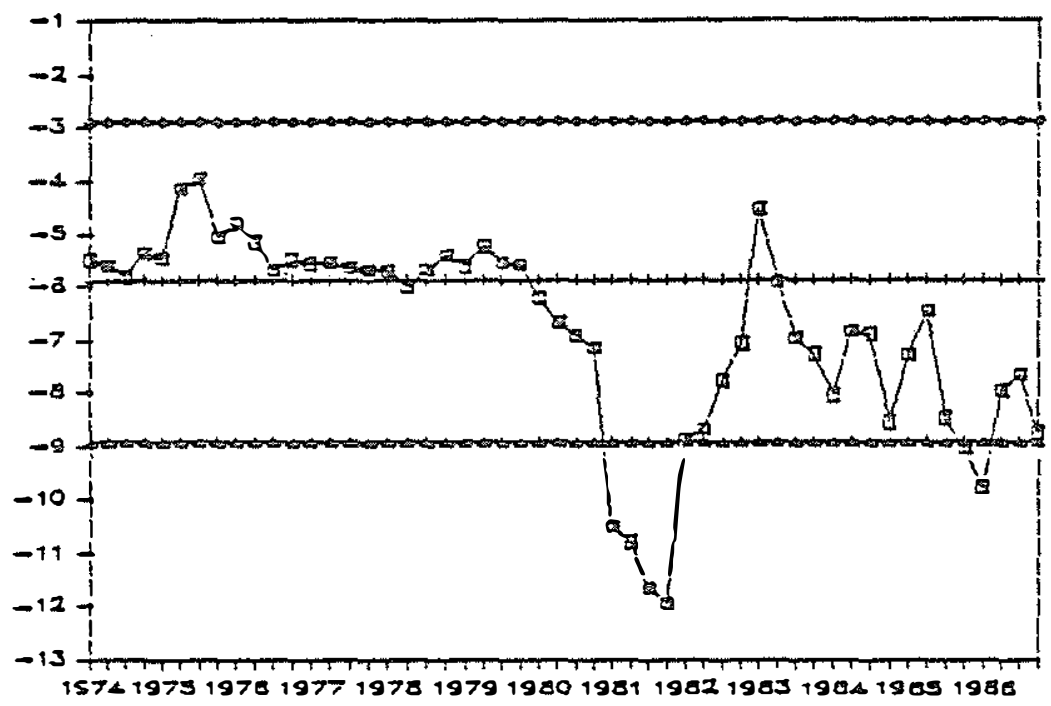

Gráfico 4

ELASTICIDAD INGRESO DE LA DEMANDA

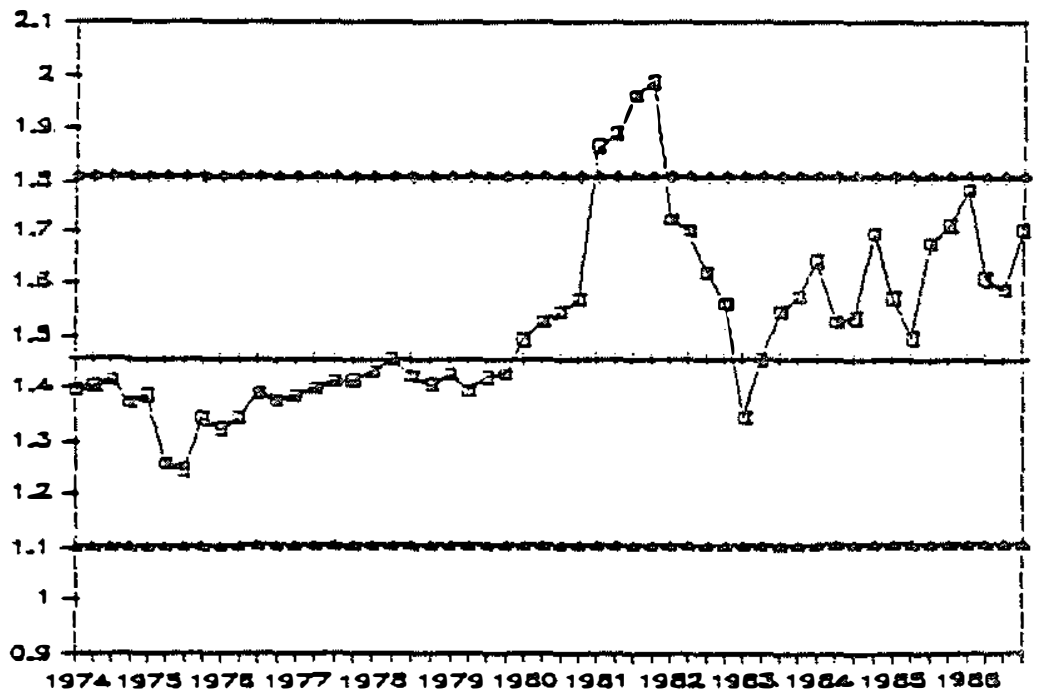

a katman $\quad$ man 
Gráfico 1

\section{SEMI-ELASTICIDAD COSTO}

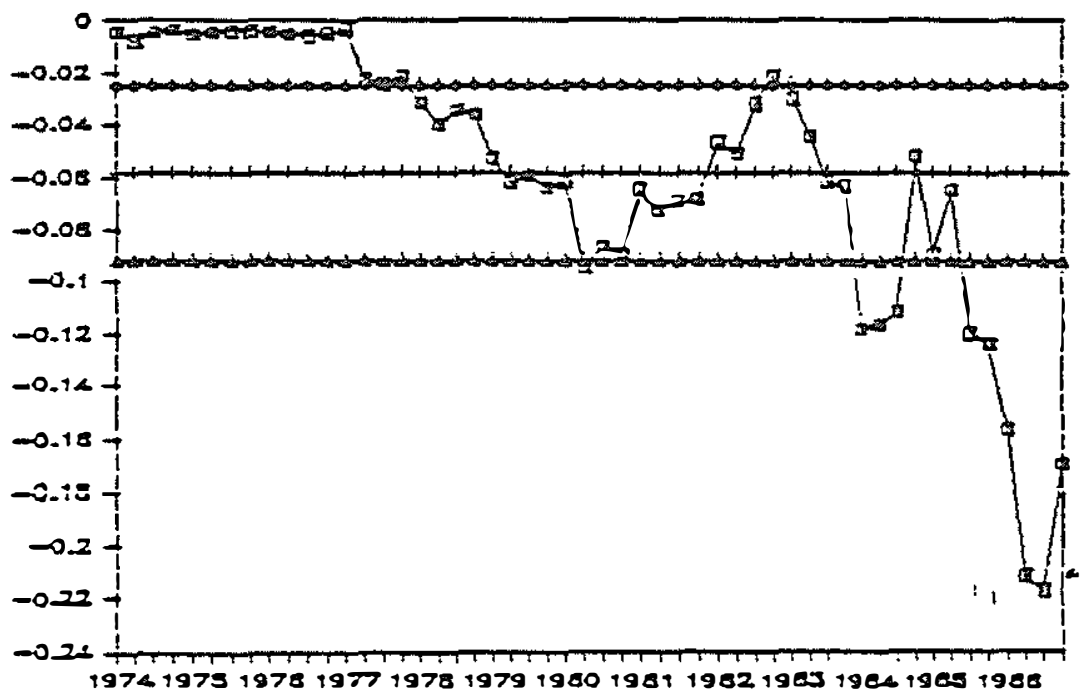

Gráfico 2

ELASTICIDAD COSTO DE LA OEMANDA

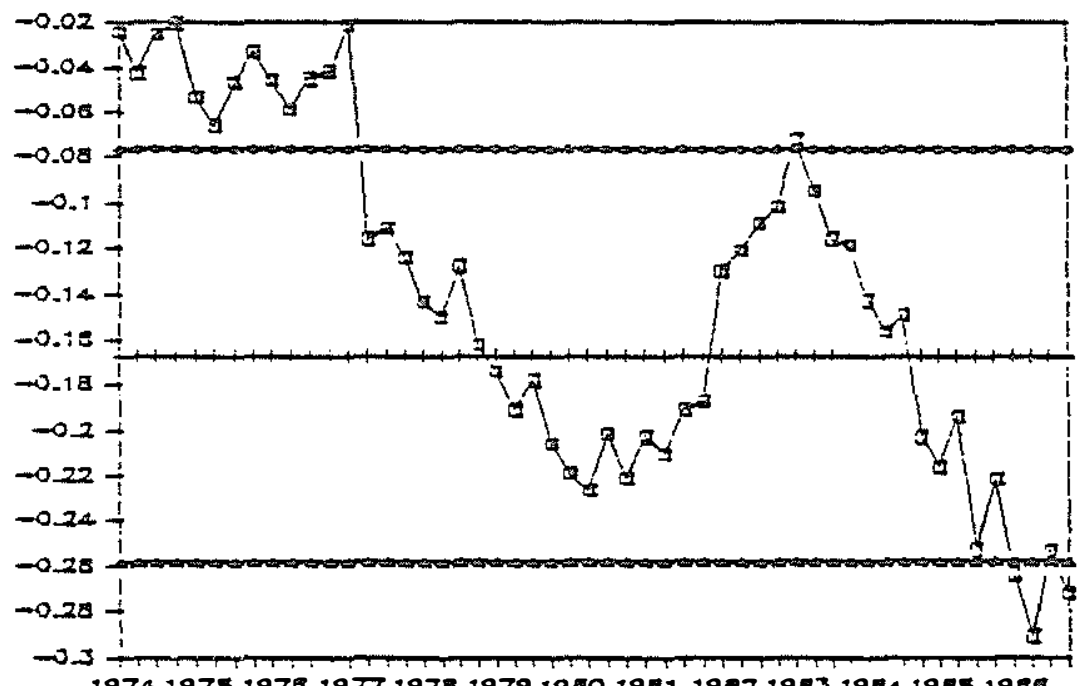

1974197519781977197819791980198119221983198419851986

a kaiman + mes 
te el resto del año. Estas reiteradas devaluaciones llevan a un fuerte deterioro de la posición patrimonial de los endeudados en dólares, y el sistema financiero ve comprometida su solvencia, la cual se agrava por una fuerte disminución en el valor de los activos y un aumento en el valor real de la deuda del sistema. Todo esto, junto a las al tas tasas de interés, termina por dibujar el perfil de la crisis financiera, la cual "obliga" a la autoridad a adoptar una série de medidas que terminan con la intervención bancaria, en enero de $1983^{22}$. En este periodo, se observa una creciente y generalizada pérdida de confianza de los agentes económicos en las reglas de la política económica, lo cual crea incentivos adicionales para invertir (recursos reales) en un mejor set de información, que les permita proyectar, de mejor forma, la evolución futura de estas variables y anticiparse a ellas. ( iv ) Post-1984, comienza un lento proceso de recuperación, favorecido en parte por un mejoramiento parcial de las condiciones externas. Este es un periodo caracterizado por cambios significativos en la institucionalidad del mercado monetario y financiero chileno, y en el que la autonomía en el diseño y manejo de la política económica ha estado limitado por los compromisos contraídos con el FMI.

Los resultados obtenidos hasta el momento se pueden sintetizar de la siguiente forma:

i) La constante presenta un comportamiento bastante estable pre-1981 y luego tiende a fluctuar erráticamente, aún cuando su variabilidad es significativa (de acuerdo a este test) durante 1981 y en 1986.1. Esta evolución nos presentaría dos mercados monetarios bastante distintos pre y post-1981. Hasta 1979 las estimaciones del FK son bastante parecidas a la estimación base, bajo el supuesto a priori de parámetros constantes. A partir de 1980 la estimación por MCO. de -5.896 , tiende siempre a subestimar (en valor absoluto), lo que efectivamente ocurre con este coeficiente. La constante captura de buena forma la intervención bancaria de enero de 1983 y la creciente inestabilidad a partir de 1982. En el primer trimestre de 1983 la demanda se transladaria (movimiento puntual) un $36,7 \%$.

A partir de 1982 ésta muestra un comportamiento errático, variando entre $-6,83$ y $-9,78$. Esto podría ser una respuesta a la creciente inestabilidad, en el contexto macroeconómico, a los contínuos cambios de las reglas de política o a los desarrollos tecnológicos observados en el mercado financiero.

ii) Con respecto a la evolución de la elasticidad ingreso, se puede observar que ésta tiene un comportamiento bastante parecido al exhi-

\footnotetext{
${ }^{22}$ Esta, entre otras graves consecuencias, arrastó al Estado chileno a garantir amortizaciones de la deuda financiera privada externa para 1983 y 84 . Un análisis detallado de la anatom la de la crisis financiera chilena de ese perfodo se encuentra en Larraín (1985).
} 
bido por el producto geográfico bruto a precios constantes y por M1 $r \in a l$. Se observa que en el tramo descendente del ciclo esta elasticidad țiende a disminuir, y lo contrario ocurre en la fase de recuperación ${ }^{23}$. En períodos de crecimiento relativamente "estable" del producto y de los saldos monetarios reales, la elasticidad ingreso muestra un comportamiento bastante estable (1976-80), y en períodos de grandes fluctuaciones de la tasa de crecimiento del stock monetario real y del producto, esta elasticidad tiende a variar erráticamente (post-1984).

Por otra parte, la diferencia entre el patrón de evolución pre y post-1981 podría indicar que el producto corriente dejó de ser la variable proxi relevante para representar los efectos ingreso (o riqueza) que operarían sobre la demanda por dinero. La creciente inestabilidad en el contexto macroeconómico y en el manejo de la política macro no permitirían que el producto corriente fuese un buen reflejo de la restricción, a largo plazo, que visualizan los demandantes de este agregado, y con la cual proyectan sus tenencias de saldos monetarios en el tiempo (con fines de transacción) ${ }^{24,25}$.

El ambiente de "triunfalismo" reinante en la economía chilena durante, los dos primeros años de esta década, explicaria en buena medida el aumento impresionante observado en dicha elasticidad en esos años. Esta sube, en un año, de 1,57 a 1,99. Es decir, el aparente boom que vivió Chile a comienzos de los ochenta, habría llevado a que los agentes confundieran el aumento observado en el ingreso corriente con un aumento en el ingreso permanente, lo cual habría inducido a un fuerte aumento en la elasticidad ingreso de la demanda por dinero con fines de transacción (ver Barandiarán, 1983).

Cabe mencionar que durante el periodo 1977 y 1980.1 esta elasticidad muestra un comportamiento muy estable, fluctuando en torno a 1,42. A partir del año 1981 ésta se torna muy inestable. Por su parte, la elasticidad ingreso obtenida por la técnica. tradicional de los MCO, de 1,45, para todo el período, tiende a sobrestimar lo ocurrido hasta 1980 y luego lo subestima, con la sola excepción de 1983.1, trimestre en el cual se produce la intervención bancaria por parte de la autoridad económica.

iii) Con respecto a la evolución de la elasticidad y de la semi-

${ }^{23}$ Esto tambiên podría indicar un posible error de especificación en la forma funcional adoptada en la ecuación ( 2 ), debido a la existencia de no-linealidad sn sus argumentos. Esto será sometido a un test más adelante $y$ un análisis detailado de ello se encuentra en Labán (1987a).

\footnotetext{
${ }^{24}$ Esta afirmación se puede demonstrar utilizando el teorema de "extracción de señales".

25 Para ser consistentes con el marco teórico planteado en la sección 2 se debe reconocer una restricción presupuestaria (intertemporal) dinámica, que permita considerar las proyecciones que realizan los agentes económicos sobre el marco macroeconómico futuro que enfrentarán. Ver Sargent (1981).
} 
- elasticidad costo de dicha demanda, aparentemente la disminución observada en la tasa de inflación y en la tasa de interés nominal contribuyó en gran medida al aumento observado en tales parámetros, en el periodo 1977-81. La elasticidad costo aumentó, en valor absoluto, de $-0,021$ a $-0,22$, en dicho período. Pero existian varios otros factores que habrian influido en dicho aumento. Primero, el desarrollo del mercado de capitales a muy corto plazo permitió el surgimiento de un mayor número y de mejores sustitutos para M1, los cuales, además de compartir con este último una gran liquidez, ofrecian una al ta tasa de rentabilidad ${ }^{26}$. Esto se vio facilitado por el acelerado proceso de "innovación financiera".

algunos instrumentos se hicieran más líquidos; facilitó y abarató el manejo de saldos en efectivo por parte de los agentes económicos; disminuyó el costo de transacción entre efectivo y estos distintos instrumentos y abrió la posibilidad para invertir los excesos de liquidez por periodos muy cortos de tiempo, a tasas no depreciables. También habria que mencionar el proceso de desregulación del sistema financiero y de privatización de la banca comercial iniciado el segundo trimestre de 1975. Por úl timo, y no por ello menos importante, la liberalización comercial y financiera con el exterior posibilitó que los agentes económicos pudieran mantener en sus portafolios instrumentos distintos a los ofrecidos en el mercado doméstico. Esto sugerixia la necesidad de considerar explícitamente las posibilidades de "slistitución de moneda", al igual que los factores que afectan esta decisión en el diseño e implementación de la política monetaria. De esta forma, la caída observada en la elasticidad interés durante 1982 y 1983, de un valor cercano a $-0,22$, durante 1981 , a $-0,09$, en el segundo trimestre de 1983, podría estar asociada a que la crisis finaciera internacional limitó la cantidad de sustitutos externos de M1.

El que esta mayor sensibilidad a precio de la demanda por dinero se haya dado junto con una creciente variabilidad en el valor de dicha elasticidad se podría explicar por un entorno de creciente incertidumbre generalizada ${ }^{27}$, por las importantes $y$ abruptas transformaciones que se dieron tanto en las condiciones macroeconómicas como en el manejo de la política económica, y por las distintas formas en que los demandantes de liquidez visualizan estos cambios en el contexto prevaleciente.

Post-1984, esta creciente incertidumbre se habria manifestado

${ }^{26}$ Ver Arellano (1983).

${ }^{27}$ Un buen indicador de la incertidumbre generalizada (de corto plazo) sobre la evolución de las distintas herramientas de la política macroeconómica, en esos años, es la encuesta mensual llevada a cabo por INECON.

${ }^{28}$ La razón papeles públicos M1 aumenta de 0.9 en promedio en 1983, a 1,83, para 1986. 
por una fuerte sustitución de $\mathbf{M I}$ para papeles públicos, debido a la confianza de los agentes en la recuperabilidad de la deuda oficial ${ }^{2} 8$. Por otra parte, la expansión del gasto agregado en 1984 habría alèntado expectativas de devaluación y de alza arancelaria, e inducido a una fuerte sustitución desde dinero hacia bienes transables y hacia dinero y activos internacionales, dado un contexto de restricciones al acceso de moneda extranjera ${ }^{29}$. Todo esto, junto al establecimiento de una tasa "sugerida" de captación desde mediados de diciembre de 1982, sugeriria que la tasa de interés usada como proxy del costo esperado de mantener dinero habría dejado de cumplir esta función. requiriéndose una definición más amplia, que, entre otras variables, considerara las expectativas de devaluación, para capturar mejor el verdadero costo esperado.

Por su parte, la elasticidad costo estimada por MCO, de $-0,167$, sobrestima la elasticidad encontrada por el FK para los periodos $1974.1,-78.4$ y $1982.1,-84.3$, y lo contrario ocurre en el resto del período muestral. Por último, la semi-elasticidad interés obtenida por MCO es de $-0,058$, la cual sobrestima la obtenida a través del FK durante 1974.1, -79.2 y durante 1982.1, -83.2. Lo contrario ocurriría en el resto del período.

Todo este periodo (1974-86) se caracteriza por un uso discrecional de los distintos instrumentos de la política económica (aún cuando con distinta intensidad), lo cual podría, por lo menos a nivel teórico, haber causado las fluctuaciones observadas en los coeficientes de la demanda por dinero ${ }^{3} 0$.

Hay que destacar el deteriorado nivel presentado por M1 real tras la brusca caída registrada por éste en 1984.1, el cual se habría mantenido hasta el segundo trimestre de 1986. Esto llama la atención a la significativa reducción en las tasas de interés junto a la considerable recuperación mostrada por el nivel de producto, de 1985 a la fecha, los cuales serian concordantes con una evolución más favorable de la demanda por dinero que la exhibida ${ }^{3}$.

Por otra parte, con el fin de analizar si las fluctuaciones obtenidas en los coeficientes de nuestra función se deben a razones ajenas a los cambios que se dan en la estructura macroeconómica y/o en el manejo de la política económica, llevamos a cabo dos distintos tests, que ${ }^{29}$ Esta hipótesis se ve alentada por el pobre desempeño exhibido por la balanza comercial, aún tras la fuerte devaluación y alza arancelaria de septiembre de 1984.

${ }^{30}$ Un ejemplo ilustrativo de la relación que se da entre un uso discrecional de la politica monetaria, el esfuerzo de los agentes económicos en proyectar estos cambios y la estabilidad de los coeficientes de un modelo econométrico, se puede encontrar en Labán (1987b).

${ }^{31}$ Para un desarrollo de las principales hipótesis que explicarian esta evolución, ver Matte y Rojas (1986). 
Gráfico 5

EVOLIJCION MI REAL

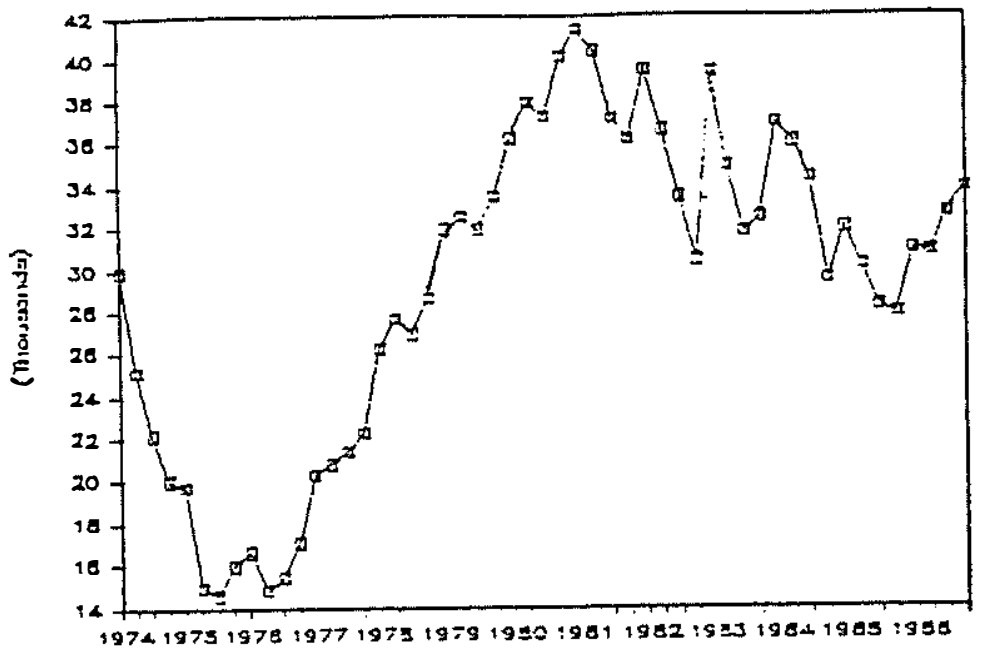

Gráfico 6

EVOLUCION PGE REAL

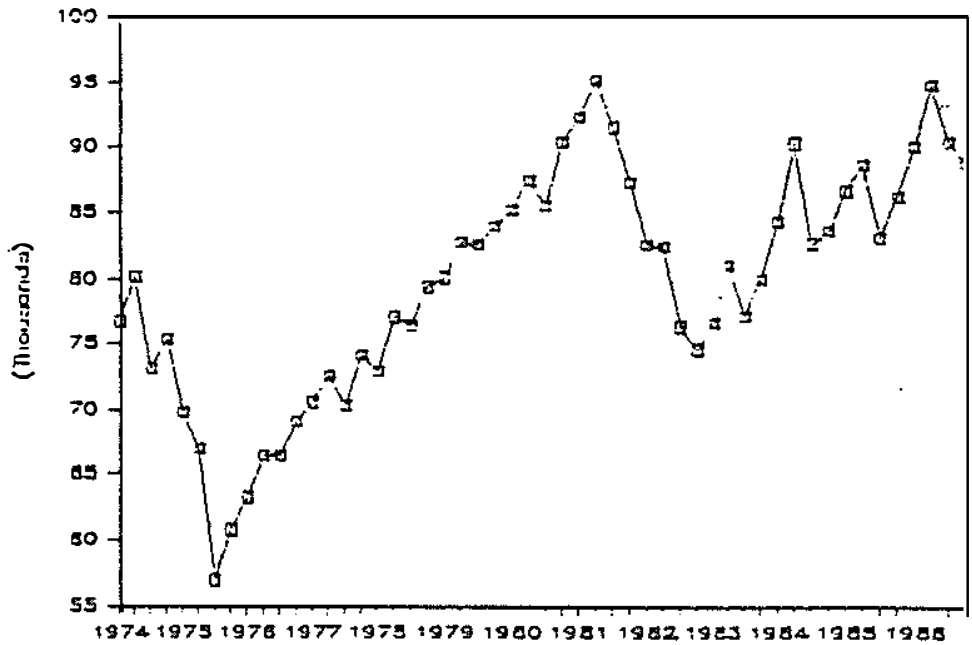


Gráfico $\%$

EVOLUCION TASA DE INTERES NOMINAL

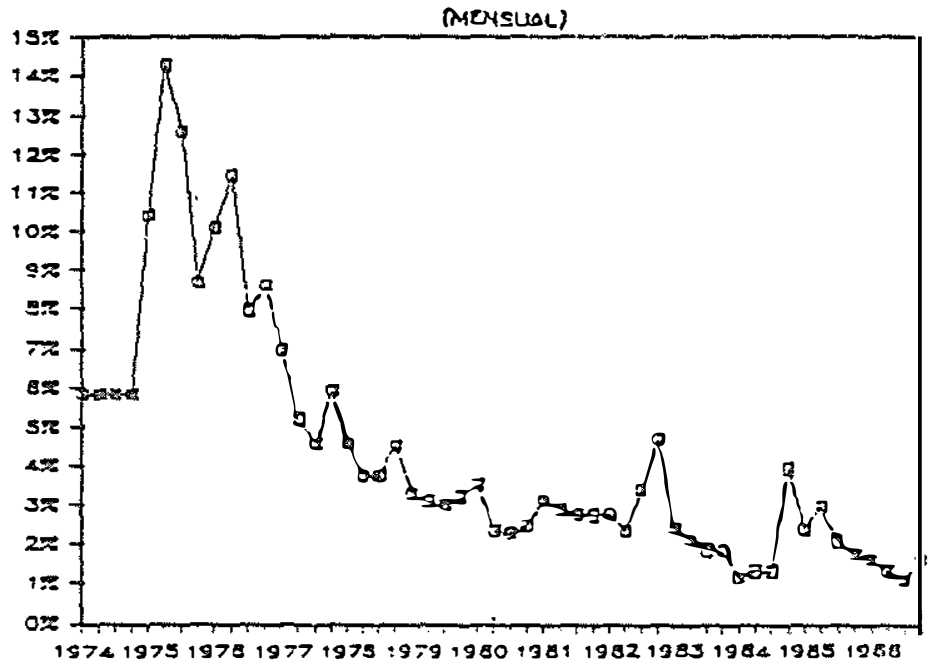

Gráfico 8

EVOLUCION TASA DE IMIFLAC:ON

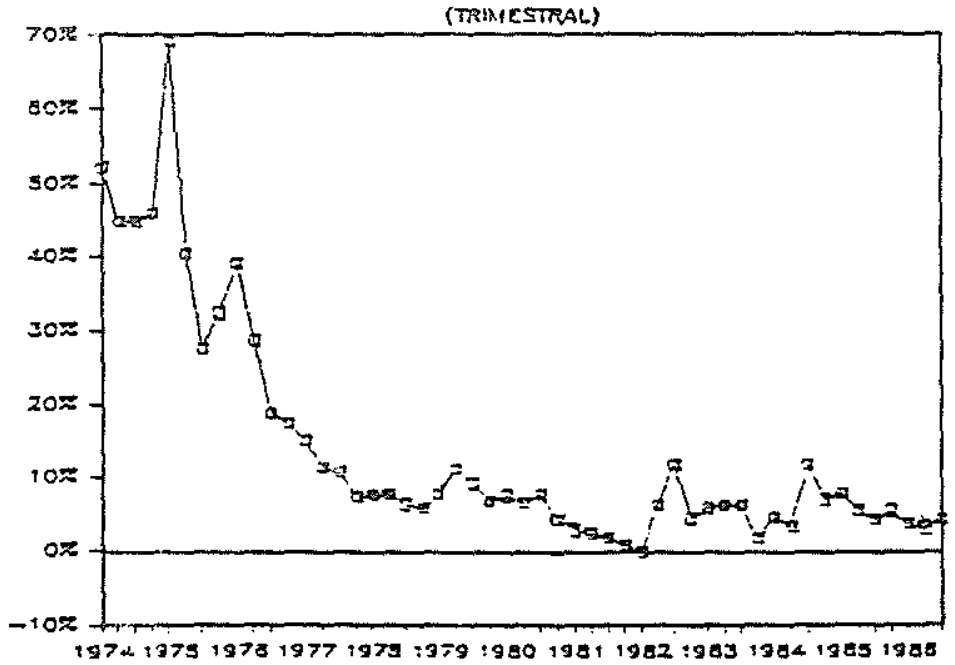


nos permiten descartar la posibilidad de que esta variabilidad se deba a motivos no económicos ${ }^{32}$.

Primero, desarrollamos un test similar al planteado por Watson $(1983)^{33}$, que nos permite averiguar si el FK es capaz de proveer estimadores insesgados y de varianza mínima (eficientes) de los coeficientes desconocidos de un modelo lineal clásico de regresion ${ }^{34}$.

Si el FK es óptimo, la secuencia de errores de predicción "un paso hacia adelante", que son computados durante el proceso recursivo, serán normal e independientemente distribuídos, con media cero y varianza $\sigma_{\mathbf{u}}^{2} \mathrm{f}_{\mathrm{t}}$, la cual también es calculada durante el proceso de fil tración. Es decir,

$$
e_{t} \sim N\left(0, o_{u}^{2} f_{t}\right) \quad t=1,2, \ldots, N
$$

Estandarizando esta distribución normal se obtiene que,

$$
\tilde{e}_{\mathrm{t}}=\mathrm{e}_{\mathrm{t}} / \sigma_{\mathrm{u}}^{2} \mathrm{f}_{\mathrm{t}}^{1 / 2} \sim \mathrm{N}(0,1)
$$

Se puede testear la propiedad de independencia de estos errores estandarizados usando la razón de Von Neuman "Modificada" (RVNM) ${ }^{35}$, definida como,

$$
\operatorname{RVNM}=\frac{\sum_{t=k+2}^{T}\left(\tilde{e}_{t}-\tilde{e}_{t-1}\right)^{2} /(n-k-1)}{\sum_{t=k+1}^{T}\left(\tilde{e}_{t}\right)^{2} /(n-k)} \nabla_{t}
$$

${ }^{32}$ Estos motivos están asociados a problemas de agregación y errores de especificación, entre otros. Ver Engle e Watson (1985) y Maddala (1977). Aún cuando estos tests no excluyen todas las posibilidades, nos permiten apoyar las conclusiones a que hemos llegado en este trabajo.

${ }^{33}$ Que también es válido para muestras pequeñas, pero que adicionalmente nos permite evitar zonas inconclusas. Este test es desarrollado en Labán (1987a), siguiendo la literatura sobre resíduos recursivos. Ver Phillips y Harvey (1974).

${ }^{34}$ Watson (1983) denomina a este test como test de optimalidad del FK, y demuestra que el FK ofrece estimadores óptimos cada vez que los MCO lo hagan e incluso en algunos casos en que estos últimos ofrezcan estimaciones sub-óptimas.

${ }^{35}$ Esta razón modificada asume que ẽ $=0$, si el $F K$ es óptimo. Donde e representa a la media de los errores de predición estandarizados. Es decir,

$$
\tilde{e}=\sum_{t=k+1}^{T} e_{t} /(n-k) .
$$


con ( $\mathbf{n}-\mathbf{k}$ ) grados de libertad. Si la propiedad de independencia no se puede verificar, el fil tro no entregará predictores óptimos.

Este test tiene la debilidad de sólo permitir testear las siguientes alternativas,

$$
\begin{aligned}
& H_{0}=\tilde{e}_{t} \sim \text { ii dN }(0,1) \\
& H_{1}=\tilde{e}_{t}
\end{aligned}
$$

sigue un proceso autorregresivo de primer orden, es decir, se genera por un proceso $A R(1)$.

Es bastante obvio qúe estas dos alternativas no agotan todas las posibles distribuciones de $\tilde{e}_{t}$. Sin embargo, si la hipótesis nula no se puede rechazar, no podremos descartar la idea de que el FK nos ofrece predicciones óptimas.

Este test lo llevamos a cabo al final del proceso de predicción y actualización con 48 grados de libertad ${ }^{36}$ y con un intervalo de confianza de un $90 \%$ (test de dos colas). El límite crítico inferior es $\left(d_{1}=1,525\right)$ y el superior es $\left(d_{u}=2,477\right)^{3}$ ? Los resultados de la aplicación de este test se pueden encontrar en el cuadro 1. Esto nos permite concluir que no es posible rechazar la hipótesis nula de independencia, ya que el valor estimado es $d^{*}=1,612$.

Un segundo problema de este test es que los errores de predicción "um paso hacia adelante" pueden estar sesgados. Es decir, mostrar un patrón de sub o sobrepredicción sistemática. Esto significa que podemos estar frente a un error de especificación funcional de la ecuación ( 2 ), en cuyo caso parte de la variabilidad experimentada por los coeficientes podría estar asociada a la existencia de no-linealidad en los argumentos de esta ecuación.

Al considerar la forma lineal, cuando la verdadera forma (modelo correcto) es una no-lineal, estaremos excluyendo variables relevantes del análisis, sesgando nuestros coeficientes. Esto tiene implicancias sobre las inferencias que se derivan del análisis de estimación, aún cuando no afecta el poder predictivo de nuestro modelo ${ }^{38}$. Para testear la significancia de este sesgo uno puede usar el resultado de que si el $\mathbf{F K}$ es óptimo y los $e_{\mathbf{t}}$ tienen las propiedades indicadas, entonces,

$$
\tau=\tilde{e} / \hat{\sigma}_{e} \sim t(n-1)
$$

donde.

${ }^{36}$ Esto se debe a que $n=52, k=3$ y se pierde un grado de libertad al partir prediciendo desde el segundo trimestre de 1974.

${ }^{37}$ Ver la tabla 8-7 (The Modified Von Neuman Ration), Johnston (1985), pág. 559. Ver Labán (1987a) para un desarrollo más formal de este test.

${ }^{38}$ Ver Johnston (1985). 


$$
\hat{\sigma}_{\mathrm{e}}^{2}=\sum_{\mathrm{t}=\mathrm{k}+1}^{\mathrm{N}} \tilde{\mathrm{e}}_{\mathrm{t}}^{2} /(n-k)
$$

es un estimador insesgado de la varianza de $\tilde{\mathbf{e}}_{\mathrm{t}}{ }^{39}$. En este caso, las hipótesis nula y alternativa a testear serían,

$$
\begin{array}{llll}
H_{0}=E\left(e_{t}\right)=0 & \nabla t & \text { (insesgamiento) } \\
H_{1}=E\left(e_{t}\right) \neq 0 & \nabla_{t} & \text { (sesgamiento) }
\end{array}
$$

El no cumplimiento de cualquiera de estos dos tests nos llevaría a rechazar la hipótesis nula de optimilidad. Un rechazo del test de Student nos indicaria que las estimaciones del filtro están sesgadas (sesgo de especificación).

Los resultados del segundo test también se encuentran en el cuadro 1. En éste se observa que no se puede rechazar que la forma funcional correcta sea lineal.

En resumen, los resultados de ambos tests nos permiten apoyar la idea de que las fluctuaciones observadas en el valor de los coeficientes se deben a cambios ocurridos en el contexto macroeconómico y/o a un uso discrecional de los instrumentos de la política económica. Por otra parte, nos permitiría afirmar que la aparen te correlación positiva existente en tre la evolución de la elasticidad ingreso y la evolución del producto real, y negativa entre la elasticidad interés con la evolución de la tasa de interés nominal, se debe a que la evolución de ambas variables están condicionadas, de alguna u otra forma a los cambios ocurridos en el marco económico e institucional y en las reglas de política aplicadas.

Por último, con respecto al análisis de sensibilidad de los resultados obtenidos - ver gráficos 9 a 12 - se puede concluir que éstos no están condicionados al valor de $\theta$ y/o $T$ seleccionados, ya que el patrón de evolución mostrado por ambas elasticidades ${ }^{40}$ mantiene sus tendencias y quiebras a través del tiempo.

\section{2 - Capacidad predictiva fuera de la muestra}

El problema de predicción es el de combinar información pasada con información corriente, para generar una distribución pro-

\footnotetext{
${ }^{39}$ Para la especificación de este test y su aplicación a los residuos recursivos (errores de pronóstico estandarizados) ver Harvey y Collier (1977).

${ }^{40}$ No se han presentado los gráficos del análisis de sensibilidad de la constante ni de la semi-elasticidad interés, pues ellas muestran un resultado similar y ocupan espacio valioso.
} 
Gráfico 9

\section{ELASTICIDAD INGPESO DEMANANA POR DINERO}

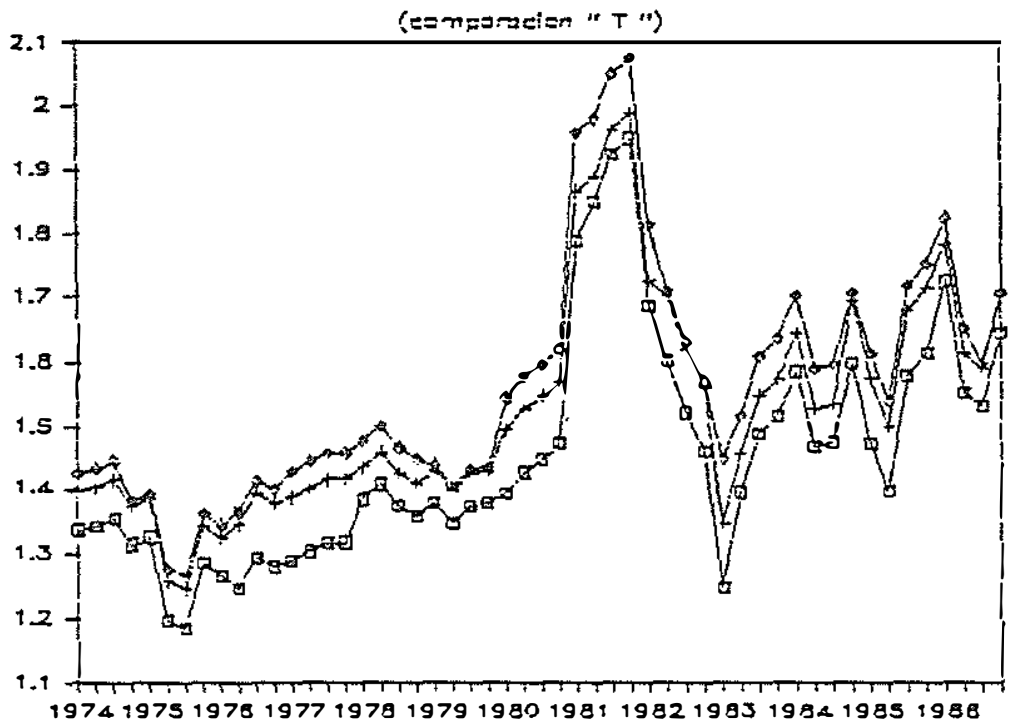

Gráfico 10

ELASTICIDAD COSTO DEMANANA FOP DINERO

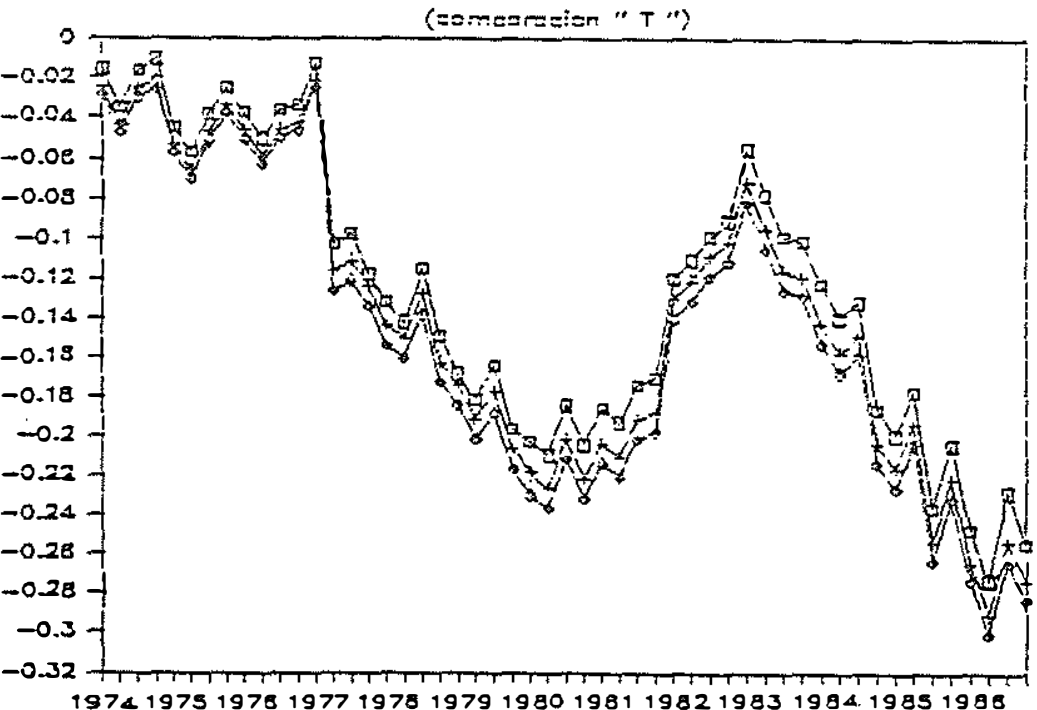

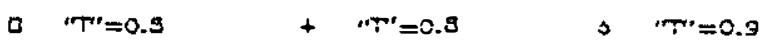




\section{Gráfico 11}

ELASTICIDAD INGRESO DEMANDA POR DINERO

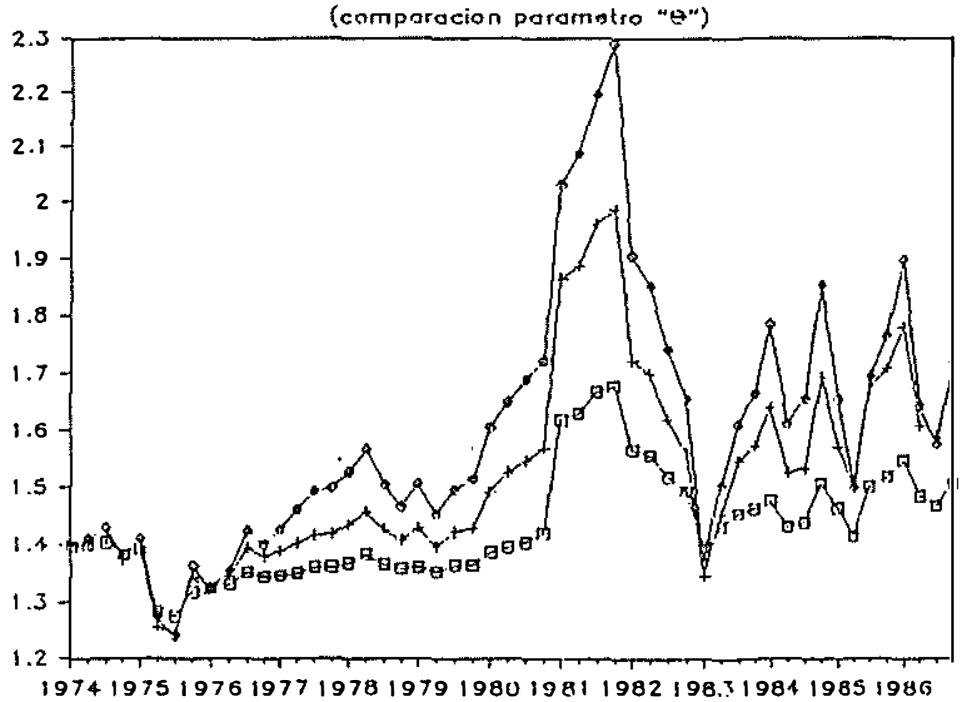

Gráfico 12

ELASTICIDAD COSTO DEMANDA POR DINERO

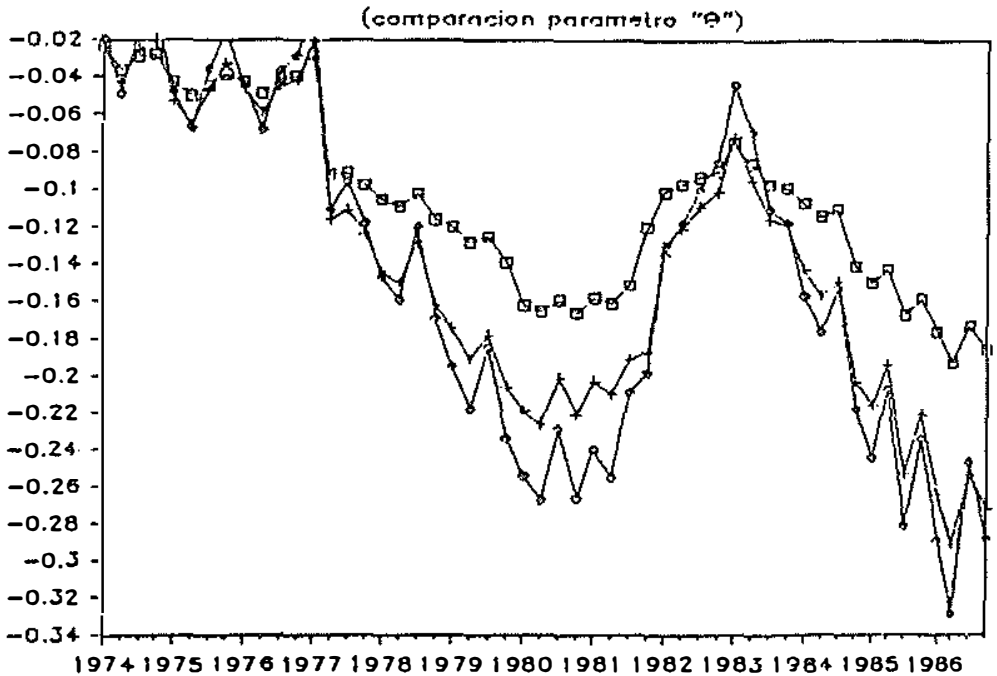

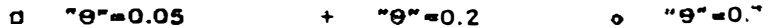


Cuadro 1

Test de Optimalidad del Filtro de Kalman

\begin{tabular}{lccc}
\hline Test & $\begin{array}{c}\text { Valor } \\
\text { Estimado }\end{array}$ & \multicolumn{2}{c}{ Intervalos de Confianza } \\
\hline $\begin{array}{l}\text { Razon de Von Neuman } \\
\text { Modificada }\end{array}$ & 1,612 & 1,525 & Superior \\
\hline $\begin{array}{l}\text { Test de Harvey y } \\
\text { Collier }\end{array}$ & 0,145 & $-1,676$ & 1,676 \\
\hline
\end{tabular}

babilística de determinados eventos futuros. Estas predicciones deben ser lo más confiables posibles, especialmente cuando son usadas como base para guiar la política económica. Dentro de este contexto, la tarea de realizar proyecciones relativamente afinadas acerca del comportamiento de la demanda por saldos monetarios constituye, para las autoridades monetarias, un aspecto central dentro de sus funciones. Esta función se hace aún más crítica cuando la autoridad monetaria enfrenta restricciones muy severas al manejo de su politica, como las que actualmente enfrentan gran parte de los países del cono sur, y en particular Chile.

La proyección de los deseos de liquidez de una economía puede, eventualmente, ser una tarea sencilla, mientras la cantidad y calidad de los activos que proveen liquidez sea relativamente constanie y la economía se desenvuelva en un contexto estable. Dicha tarea se ve dificultada considerablemente en períodos de contínuos cambios en las reglas de política y de profundas transformaciones en el contexto macroeconómico, como el vivivo por Chile en el período considerado. Estos canbios contribuyen a un creciente grado de incertidumbre sobre la estructura filtura de la economia. En dichos periodos, uno esperaria que el FK, que captura de mejor forma los elementos estocásticos de la económia, superase significativamente la capacidad que tienen las técnicas estáticas tradicionales para generar una realista distribución de probabilidades de eventos económicos futuros $y$, en particular, de la demanda futura por liquidez.

En resumen, al menos a nivel intuitivo, uno debiera esperar que una técnica dinámica de estimación, que relajase el supuesto a priori de constancia de los coeficientes, como es el FK, ofreciese predicciones más certeras de la evolución posible de determinadas variables o eventos fiuturos, que una técnica estática, como la de los MCO. Las razones para esto las podemos sintetizar como sigue:

i) El modelo del FK permite considerar de mejor forma la característica cambiante del contexto macroeconómico, en términos 
generales, la cual es incorporada en el proceso de estimación y predicción;

ii) permite realizar predicciones relativamente afinadas con un pequeño número de observaciones - al combinar información a priori con las observaciones actuales -, perdiendo importancia la discusión sobre los grados de libertad disponible; y

iii) posibilita la actualización (revisión) de los coeficientes y predicciones al integrarse al set de información una nueva observación. Esta técnica permite descontar, de una forma óptima, el peso que tienen las observaciones pasadas sobre las estimaciones y predicciones actuales.

En lo que sigue compararemos el poder predictivo relativo de la técnica del FK con el de las técnicas tradicionales de los MC̣O y los MCO recursivos ( MCOR ). Esta comparación la llevaremos a cabo en base a distintas medidas de certeza predictivas tradicionales, basadas en los errores de predicción. Estas medidas son: la raiz del error cuadrático medio ( RECM), error medio absoluto (EMA) y el coeficiente de desigualdad de Theil $(U)^{41}$.

Las tres medidas tendrían un valor igual a cero si la predicción fuese perfecta. Las dos primeras están definidas entre cero y uno. La tercera tendria un valor de uno con una predicción igual a aquella que supone que:-el valor de la variable dependiente no cambia, y sería mayor que la unidad si la predicción fuera menos certera que una pre. dicción simple de constancia. Con el fin de analizar si los resultados de la comparación dependen del número de pasos hacia adelante en que se hace la predicción, hacemos la comparación para predicciones desde uno hasta cuatro pasos hacia adelante.

En esta sección se desea resaltar la necesidad de que la autoridad monetaria incorpore explicitamente en su proceso de toma de decisiones este comportamiento cambiante de los agentes económicos frente a cambios en las reglas de politica (inestabilidad inducida por un uso discrecional de la polftica económica), como también el impacto que tienen sobre la demanda por dinero diversos eventos como, por ejemplo, el proceso de innovación financiera (shocks tecnológicos exógenos) y los posibles cambios en las preferencias de los individuos.

La autoridad mencionada, al ser capaz de incorporar estos hechos, podrá predecir, de mejor forma, la demanda futura por los distintos agregados monetarios que controla y asi podrá obtener resul. tados más concordantes con los objetivos que persigue con la implementación de su política. Es decir, un reconocimiento explícito de estos distintos elementos, le permitirá tener un mayor grado de control sobre sus acciones.

El análisis de comparación lo realizamos para los cuatro trimes-

${ }^{41}$ Para un análisis detallado te estas medidas, ver Fair (1984). 
tres del año 1986. Para ello reestimamos el período 1974.1, -85.4 a través del FK y de la técnica estática de los MCO. Luego proyectamos la demanda por $\mathbf{M 1}$ real de las siguientes formas: ( $\mathrm{i}$ ) usando el valor (único) de los coeficientes estimados para todo el período, bajo el supuesto de que se mantienen constantes durante el período de inferencia - proyección estática por MCO-, ( ii ) utilizando los estimadores MCO $(1974.1,-85.4)$ y reestimándolos a través de esta misma técnica cada vez que una nueva observación se hace disponible. Este métódo supone que los coeficientes varían sólo debido a que se incluyó en la muestra una nueva información, y atribuye el mismo peso a todas las observaciones pasadas sobre la estimación y predicción actual - proyección por MCOR-, ( iii) aplicando el filtro a las observaciones trimestrales de los años 1974-85. Esto significa que las observaciones más lejanas van perdiendo peso frente a las estimaciones y predicciones corrientes, y que los coeficientes también pueden variar como respuesta a los elementos de innovación. Esta técnica proporciona un mecanismo de actualización de los coeficientes que son usados en el proceso de predicción - proyección a través del FK-.

Los resultados de este proceso de predicción y comparación pueden ser encontrados en los cuadros 2 a 6 y en el gráfico 13. De estos se puede concluir que:

i) los errores de predicción reportados por las tres técnicas son considerablemente altos, alcanzando en algunos casos un $50 \%$ del valor efectivo ( MCO para 1986.2) ${ }^{42}$. Para igual trimestre el error de predicción del $\mathrm{FK}$ es cercano al $20 \%$ (su valor máximo). Para todos los pasos analizados - a través de las tres medidas - el FK ofreció predicciones más certeras que las otras dos técnicas. Los MCOR superaron las predicciones proporcionadas por los MCO. Esto indicaría que, en la medida en que seamos capaces de incorporar la estructura cambiante del entorno a nuestro análisis, obtendremos resultados más precisos. Ver cuadro 2.

ii) Al analizar el coeficiente de Theil (U) se observa que sólo en 1986.4 las técnicas del FK y de los MCOR ofrecen predicciones más correctas que las obtenidas a partir del supuesto de que el stock deseado de $\mathbf{M 1}$ real no cambia $\left(\mathbf{M 1}_{\mathbf{t}-\mathbf{1}}=\mathbf{M} \mathbf{1}_{\mathbf{t}}\right)$. Es decir, a excepción de 1986.4 y para estas dos técnicas $\mathbf{U}>\mathbf{1}$.

iii) Las desviaciones estándar del error de predicción son dema.

42 El error de predicción de M1 real se puede calcular a partir de los errores presentados en el cuadro 3 de siguiente forma,

$$
\left.(\exp )^{\mu}-1\right): M 1 \text { efectivo }=\epsilon,
$$

donde

$\mu$ error de predicción en términos logarítmicos y

$\epsilon$ error de predicción de $\mathbf{M 1}$ real. 
Cuadro 2

Errores de Predicción en niveles y como Porcentaje del Valor Efectivo (1986.1 - 1986.4)

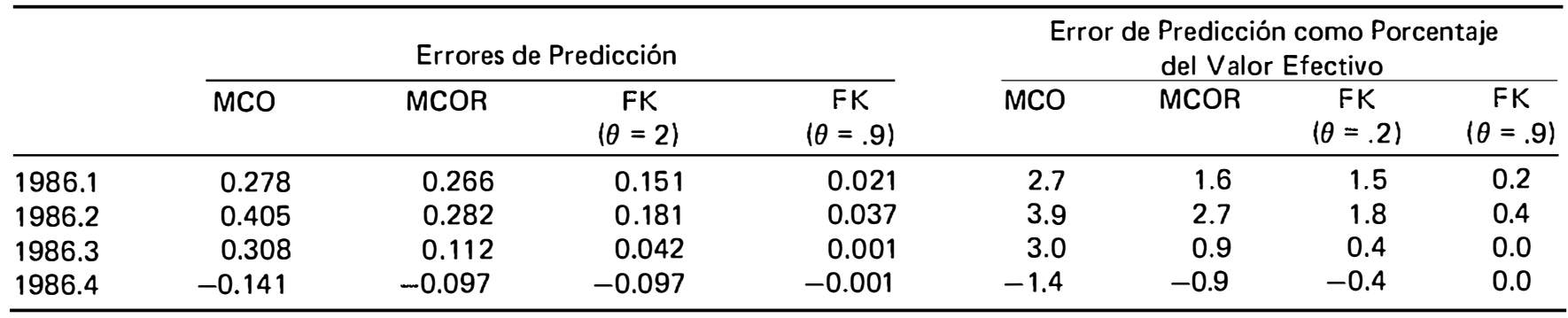




\section{Cuadro 3}

M1 Real Efectivo y Proyectado a través de distintas Técnicas Econométricas (1986.1 - 1986.4)

\begin{tabular}{lccccc} 
& Efectivo & MCO & MCOR & $\begin{array}{c}\text { FK } \\
(\theta=.2)\end{array}$ & $\begin{array}{c}\text { FK } \\
(\theta=.9)\end{array}$ \\
\hline 1986.1 & 30,864 & 40,760 & 40,288 & 35,890 & 31,519 \\
1986.1 & 30,738 & 46,071 & 40,768 & 36,844 & 31,896 \\
1986.3 & 32,560 & 44,287 & 36,422 & 33,943 & 32,592 \\
1986.2 & 33,781 & 29,340 & 30,654 & $-32,274$ & 33,747 \\
\hline
\end{tabular}

Cuadro 4

Raiz del Error Cuadrático Medio (RECM)

Para la Predicción de M1 Real (1986.1 - 1986.4)

\begin{tabular}{lcccc}
\hline \multirow{2}{*}{ Técnica } & \multicolumn{4}{c}{ Número de Pasos Hacia Adelante } \\
\cline { 2 - 5 } & 1 & 2 & 3 & 4 \\
\hline MCO & 0.278 & 0.347 & 0.335 & 0.298 \\
MCOR & 0.266 & 0.275 & 0.233 & 0.208 \\
FK $(\theta=.2)$ & 0.151 & 0.167 & 0.138 & 0.122 \\
FK $(\theta=.9)$ & 0.021 & 0.030 & 0.025 & 0.021 \\
\hline
\end{tabular}

Cuadro 5

Error Medio Absoluto (EMA) Para La Predicción de M1 Real (1986.1 - 1986.4)

\begin{tabular}{lcccc}
\hline \multirow{2}{*}{ Técnica } & \multicolumn{4}{c}{ Número de Pasos Hacia Adelante } \\
\cline { 2 - 5 } & 1 & 2 & 3 & 4 \\
\hline MCO & 0,278 & 0.341 & 0.330 & 0.283 \\
MCOR & 0.266 & 0.274 & 0.220 & 0.190 \\
FK $(\theta=.2)$ & 0.151 & 0.166 & 0.125 & 0.105 \\
FK $(\theta=.9)$ & 0.021 & 0.029 & 0.020 & 0.015 \\
\hline
\end{tabular}

Cuadro 6

Coeficiente de Desigualdad de Theil (U)

Para la Predicción de M1 Real (1986.1 - 1986.4)

\begin{tabular}{lcccc}
\hline \multirow{2}{*}{ Técnica } & \multicolumn{4}{c}{ Número.de.Pasos.HaciaAdelante } \\
\cline { 2 - 5 } & 1 & 2 & 3 & 4 \\
\hline MCO & 6.878 & 5.912 & 4.101 & 2.012 \\
MCOR & 4.559 & 3.493 & 1.840 & 0.723 \\
FK $(\theta=.2)$ & 3.009 & 2.466 & 1.172 & 0.333 \\
FK $(\theta=.9)$ & 1.116 & 0.912 & 0.632 & 0.433 \\
\hline
\end{tabular}


Gráfico 13

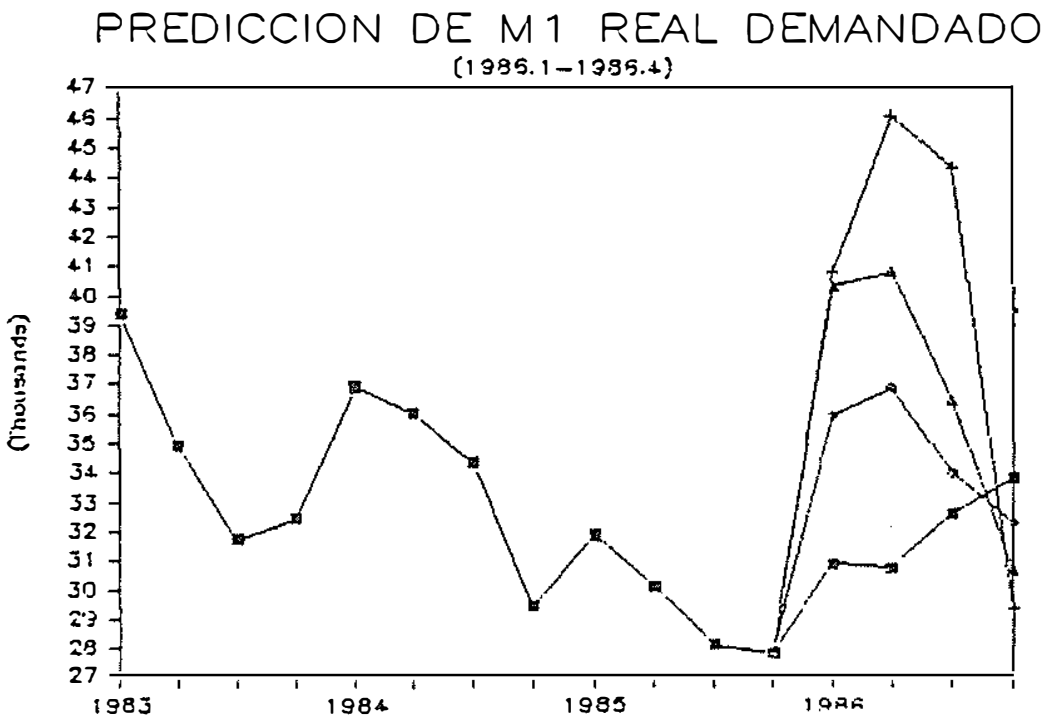

Gráfico 14

PREDICCION DE M1 REAL DEMANDADO (1980.1-1980.4.)

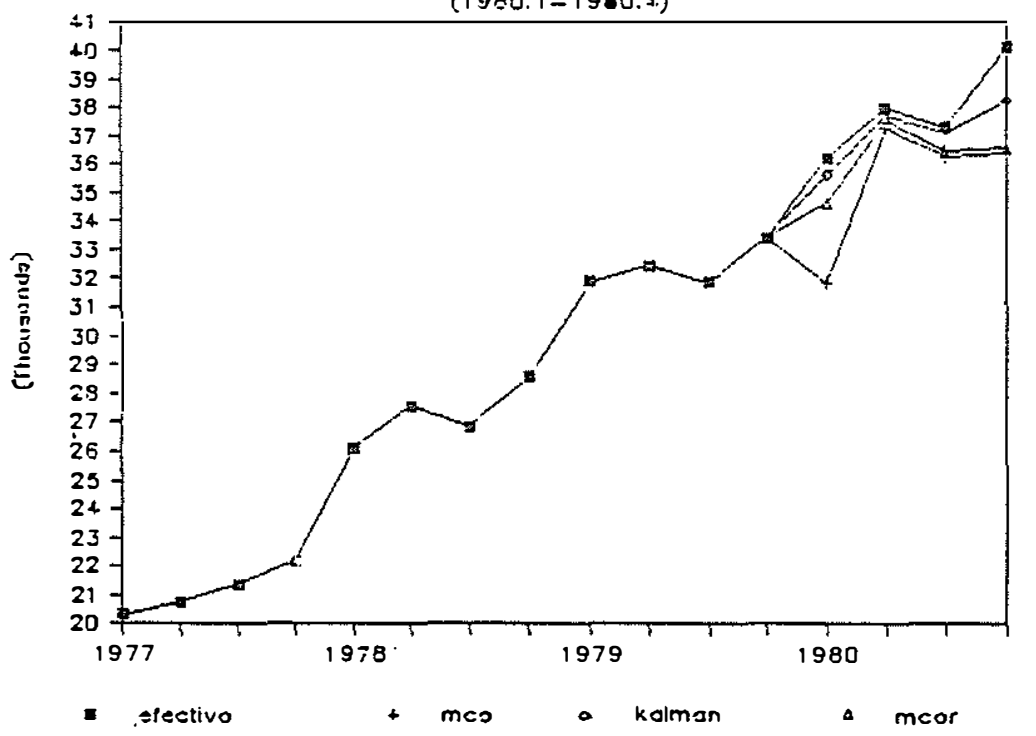


Gráfico 15

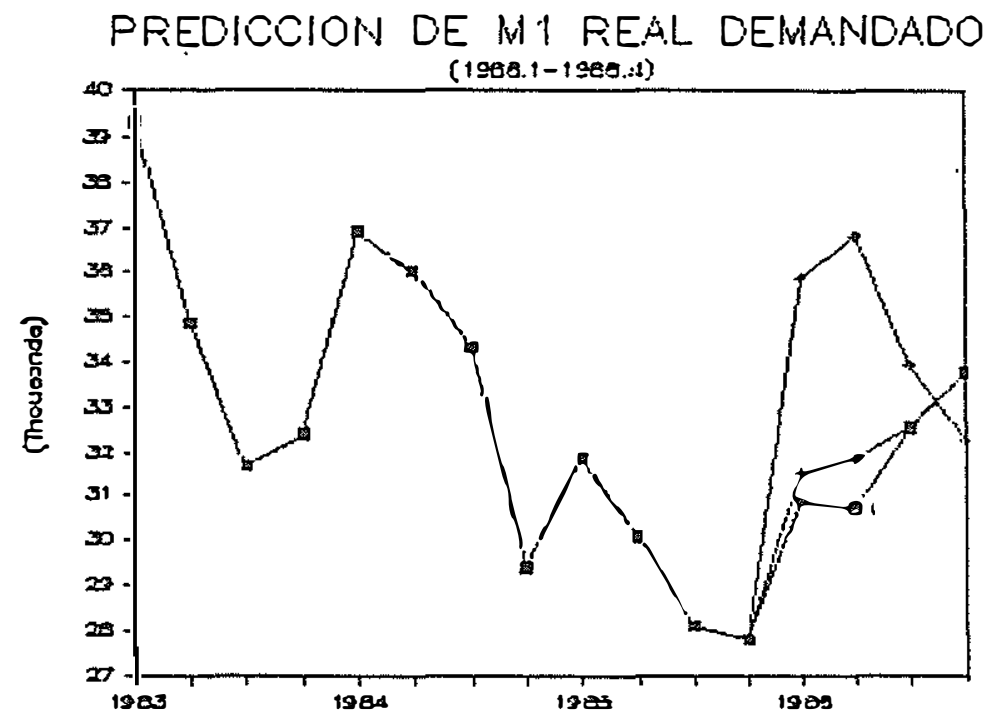

Gráfico 16

PREDICCION DE MI REAL DEMANDADO

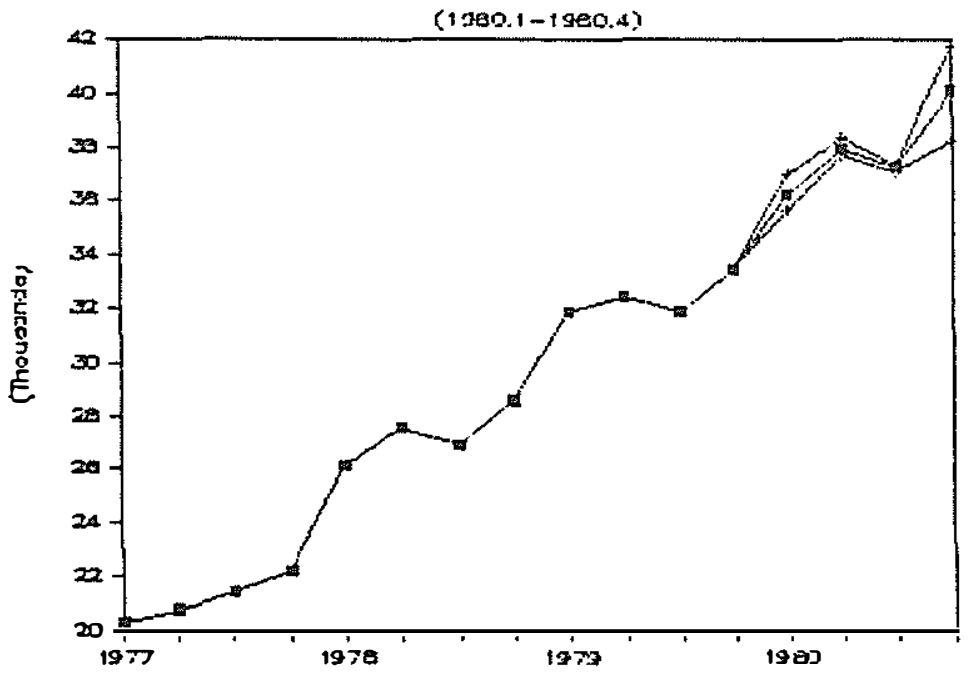

a aferive

$+\quad$ kalmanị $=0.3$ )

- kelman(0m15.2) 
siado elevadas como para intentar usar los resultados obtenidos en una programación monetaria. Es decir, tratar de acomodar la oferta monetaria a la proyeccíón de su demanda, con el fin de lograr estabilidad en uno o más de los argumentos de esta función, puede causar exactamente el efecto contrario.

iv) Con la excepción de 1986.4, nuestra ecuación ( 2 ) tiende a sobrepredecir los deseos de liquidez de la comunidad. Una posible explicación de ésto es que M1 dejó de ser el agregado monetario relevante a partir de 1984, siendo la definición de "M1 ampliado", presentada por el Banco Central de Chile, la definición relevante (ver Matte y Rojas, 1986).

De este análisis se deriva que, en un periodo tan inestable como el de 1981-86 (de acuerdo a los resultados encontrados en la sección 3.1.b), la proyección de los deseos de liquidez se convierte en una tarea extremamente dificil para la autoridad monetaria. Esto puede implicar que el manejo de la cantidad de dinero no sea una política recomendable, especialmente cuando ésta es utilizada como una herramienta de la política de estabilización de corto plazo.

El bajo poder predictivo mostrado por el $\mathbf{F K}$ en este periodo puede deberse a que, en un contexto de creciente incertidumbre y de continuos cambios en las reglas de política y en la estructura del mercado monetario y financiero, los agentes económicos muestran un comportamiento casi instantáneo, lo cual no es capturado de buena forma al asumir un $\theta=0.2$. Con el objetivo de someter a un test esta hipótesis de mal desempeño, permitiremos una respuesta más aleatoria a los cambios ya mencionados, asumiendo un $\theta=0.9$ y luego observaremos el efecto que esto tiene sobre las tres medidas de poder predictivo (ver gráfico 14).

De estos resultados se puede observar que el valor estimado para las tres medidas disminuye considerablemente y que del coeficiente $\mathbf{U}$ se desprende que el $\mathbf{F K}$ con un $\theta=0.9$ ofrece predicciones más certeras que aquellas obtenidas al suponer que el stock de dinero se mantiene constante, la cual era superior a los tres casos anteriores. Es decir, considerar como una restricción adicional al diseño de la política monetaria es ta respuesta casi instantánea de los agentes económicos, al igual que la rapidez con que cambios en el contexto macroeconómico afectan a la demanda por dinero en condiciones de gran incertidumbre, permitiria un mayor éxito a la autoridad en el manejo de su política.

Dada la característica de inestabilidad del período en que llevamos a cabo la comparación, se hace necesario comprobar si dichos resultados se mantienen en un período de "relativa" estabilidad y de relativa credibilidad en las reglas de política, como lo es el período 1977-80 en Chile. Para ello repetimos el análisis anterior, reestimando el período 1974.1-1979.4 como base, y llevando a cabo la compa1 
Cuadro 7

Errores de Predicción en Niveles y como Porcentaje del Valor Efectivo (1980.1 - 1980.4)

\begin{tabular}{|c|c|c|c|c|c|c|c|c|}
\hline & \multicolumn{4}{|c|}{ Errores de Predicción } & \multicolumn{4}{|c|}{$\begin{array}{l}\text { Error de Predicción como Porcentaje } \\
\text { Porcentaje del Valor Efectivo }\end{array}$} \\
\hline & $\overline{\mathrm{MCO}}$ & MCOR & $\begin{array}{c}\text { FK } \\
(\theta=.2)\end{array}$ & $\begin{array}{c}\mathrm{FK} \\
(\theta=.9)\end{array}$ & $\overline{\mathrm{MCO}}$ & MCOR & $\begin{array}{c}\mathrm{FK} \\
(\theta=.2)\end{array}$ & $\begin{array}{c}\mathrm{FK} \\
(\theta=.9)\end{array}$ \\
\hline 1980.1 & 0.129 & 0.045 & 0.017 & 0.021 & 1.2 & 0.4 & 0.2 & 0.2 \\
\hline 1980.2 & 0.018 & 0.010 & 0.006 & 0.011 & 0.2 & 0.1 & 0.1 & 0.1 \\
\hline 1980.3 & 0.029 & 0.023 & 0.005 & 0.002 & 0.3 & 0.2 & 0.0 & 0.0 \\
\hline 1984.3 & 0.098 & 0.092 & 0.047 & 0.041 & 0.9 & 0.9 & 0.4 & 0.4 \\
\hline
\end{tabular}


Cuadro 8

M1 Real Efectivo y Proyectado a través de

Distintas Técnicas Econométricas (1980.1 - 1980.4)

\begin{tabular}{lccccc}
\hline & Efectivo & MCO & MCOR & $\begin{array}{c}\text { FK } \\
(\theta=.2)\end{array}$ & $\begin{array}{c}\text { FK } \\
(\theta=.9)\end{array}$ \\
\hline 1980.1 & 36,223 & 31,833 & 34,640 & 35,608 & 36,992 \\
1980.2 & 37,927 & 37,254 & 37,560 & 37,699 & 38,347 \\
1980.3 & 37,269 & 36,204 & 36,438 & 37,086 & 37,344 \\
1980.4 & 40,092 & 36,341 & 36,568 & 38,252 & 41,770 \\
\hline
\end{tabular}

Cuadro 9

Raiz del Error Cuadrático Médio (RECM)

Para la Predicción de M1 Real (1980.1 - 1980.4)

\begin{tabular}{lcccc} 
& \multicolumn{4}{c}{ Número de Pasos Hacia Adelante } \\
\cline { 2 - 5 } Técnica & 1 & 2 & 3 & 4 \\
\hline MCO & 0.129 & 0.092 & 0.077 & 0.083 \\
MCOR & 0.045 & 0.032 & 0.029 & 0.053 \\
FK $(\theta=.2)$ & 0.017 & 0.013 & 0.011 & 0.025 \\
FK $(\theta=.9)$ & 0.021 & 0.017 & 0.014 & 0.024 \\
\hline
\end{tabular}

Cuadro 10

Error Médio Absoluto (EMA) para la

Predicción de M1 Real (1980.1 - 1980.4)

\begin{tabular}{lcccc}
\hline \multirow{2}{*}{ Técnica } & \multicolumn{4}{c}{ Número de Pasos Hacia Adelante } \\
\cline { 2 - 5 } & 1 & 2 & 3 & 4 \\
\hline MCO & 0.129 & 0.074. & 0.059 & 0.069 \\
MCOR & 0.045 & 0.027 & 0.026 & 0.042 \\
FK $(\theta=.2)$ & 0.017 & 0.012 & 0.009 & 0.019 \\
FK $(\theta=.9)$ & 0.021 & 0.016 & 0.011 & 0.019 \\
\hline
\end{tabular}

Cuadro 11

Coeficiente de Desigualdad de Theil (U) para

la Predicción de M1 Real (1980.1 - 1980.4)

\begin{tabular}{lcccc}
\hline \multirow{2}{*}{ Técnica } & \multicolumn{4}{c}{ Número de Pasos Hacia Adelante } \\
\cline { 2 - 5 } & 1 & 2 & 3 & 4 \\
\hline MCO & 2.341 & 1.897 & 1.327 & 0.992 \\
MCOR & 1.114 & 0.462 & 0.247 & 0.232 \\
FK $(\theta=.2)$ & 0.837 & 0.402 & 0.311 & 0.147 \\
FK $(\theta=.9)$ & 1.037 & 0.414 & 0.370 & 0.109
\end{tabular}


ración durante los cuatro trimestres de 1980. Los resultados de ésto se pueden observar en los cuadros 7 a 11 y en los gráficos 15 y 16. Las principales conclusiones que de aqui se derivan pueden sintetizarse de la siguiente forma:

i) Nuevarnente, al observar las tres medidas de poder predictivo, se puede concluir que el FK ofrece predicciones más certeras que las otras dos técnicas econométricas.

ii) El coeficiente de desigualdad de Theil nos dice que el FK siempre ofrece predicciones más precisas que aquellas derivadas del supuesto de que $M 1$ es en $t+1$ igual que en $t(U<1$ para todos los pasos). Por otra parte, este resultado también lo comparte la técnica de los MCOR, con la única excepción del primer paso. Los MCO logran un $\mathbf{U}<1$ sólo al cuarto paso, aún cuando no es significativamente distinto de uno.

iii) Los errores de predicción como porcentage del valor efectivo son considerablemente menores que los obtenidos para los cuatro trimestres del año 1986. Los MCO alcanzan un máximo de un $12 \%$ en 1980.1 , los MCOR un $8,7 \%$, el FK $(\theta=.2)$ un $4 \%$ y el FK $(\theta=$ .9) un $4.1 \%$ en 1980.4 .

iv) Las desviaciones estándares de predicción, aún cuando disminuyen significativamente, continúan siendo altas como para intentar monitorear la oferta monetaria de acuerdo a estas proyecciones. Por ejemplo, al proyectar la demanta por M1 real para 1980.2, obtenemos por MCO, con un $\alpha=10 \%$, un stock demandado que está entre 32,022 y 43,341 millones de pesos de diciembre de 1977.

v) Permitir una respuesta más instantánea de los agentes económicos y una estructura de la demanda por dinero más influída por elementos de innovación que por aquellos de tendencia [FK ( $\theta=$ .9)], en este período no tiene un efecto positivo tan claro como el observado el año 1986. Esto estaría explicado por el hecho de..que éste es un período caracterizado por una mayor estabilidad en el contexto macroeconómico y por una "mayor" credibilidad en la conducción de la politica económica, todo lo cual se resume en una evolución más de tendencia de esta función.

Por último, los resultados aquí presentados permiten plantear siguientes recomendaciones tentativas para el manejo de la política monetaria:

i) La regla de crecimien to estable del stock de dinero sólo será recomendable si la inestabilidad de su demanda es totalmente impredecible y/o causada por un uso discrecional de las reglas de política económica (inestabilidad endógena). En dicha circunstancia la sugerencia de politica sería la de un uso de reglas claras y precisas fijadas por algún tipo de arreglo institucional.

ii) Por otro lado, si dicha inestabilidad es exógena y predecible (i.e., por shocks tecnológicos o por cambios en los gustos) se reco. 
mienda que la autoridad monetaria reconozca explicitamente estos cambios en el diseño de su política. En ese caso, la demanda por dine. ro se hace estable.

Sin embargo, con el fin de determinar frente a qué contexto nos enc on tramos, se requiere llevar a cabo un análisis más concluyente de las causas precisas de esta inestabilidad. Eso escapa a los objetivos de esta investigación.

पै

5. Conclusiones

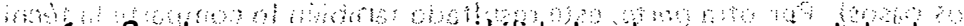

1. Se observa una gran seńsibilidad en la trayectoria de la elasticidad ingreso a la tasa de crecimiento del stock de saldos monetarios reales y del PGB. Con respecto a la evolución de la elasticidad costo se observa una creciente sensibilidad a los precios de esta función de demanda. Explicaciones tentativas tendrian que consider ar, entre otros factores, el proceso de desregulación de los mercados financieros, por el acelerado proceso de innovación financiera; la creciente integración a los mercados internacionales de bienes y activos un mayor número (y de mejor calidad) de instrumentos líquidos y renta. bles sustitutos de M1, y una creciente incertidumbre en las reglas $y$ anuncios de la política económica.

El aumento observado en la elasticidad precio de la demanda por M1 real sugeriría la creciente importancia de los elementos financieros para explicar las fluctuaciones registradas en el mercado monetario y, por lo tanto, en la economía como un todo.

2. Los resultados presentados en este trabajo permiten rechazar la hipótesis nula de estabilidad de la función de demanda por dinero entre 1974-86 para la económia chilena, siendo el periodo $1977-$ 80 un periodo "relativamente" más estable.

La evolución mostrada por la demanda por M1 nos indicaría que este agregado monetario pareciera no ser el más adecuado como criterio para guiar la politica monetaria en periodos de relativa inestabilidad económica.

3. Los resultados de aplicar los tests de óptimalidad a los errores de predicción un paso hacia adelante, ofrecidos por el FK, no nos permiten rechazar la hipótesis de que las fluctuaciones registradas en los coeficientes estén correlacionadas a los cambios observados en el contexto macroeconómico y en el manejo de la política económica.

4. Con respecto al análisis de predicción se puede concluir que:

i) El FK, al reconocer la caracteristica cambiante del contexto macroeconómico, en términos generales, ofrece mejores predicciorıes que las otras dos técnicas (estáticas), tanto en periodos de estabilidad como de inestabilidad. Intuitivamente el FK se puede descri- 
bir como un "descontador óptimo" del peso de las observaciones pasadas para obtener el mejor predictor un paso hacia adelante de la variable dependiente en cada período. Por el contrario, las otras dos técnicas le asignan igual peso a las observaciones pasadas y a la presente, al entregar las predicciones un período hacia adelante.

ii) La idea de acomodar la oferta monetaria a las proyecciones de su demanda puede no ser una regla de política recomendable en economías inestables como la chilena, en el período considerado, dada la inestabilidad y la dificultad de hacer proyecciones relativamente afinadas de esta demanda.

\section{Referências}

ARRELLANO, J. P. De la liberalización a la intervención: el rrercado de capitales en Chile, 1974-83. Colección Estudios Cieplan, 11: 5.50, Santiago, dic. 1983.

ARRAU, P. Series trimestrales del producto geográfico bruto revisado. Notas Técnicas, 89, Cieplan. Santiago. dic. 1986.

Banco Central de Chile. Sintesis Monetarias y Financieras, 1982 y dic. 1986; Boletín Mensual, n. 564, feb. 1975 y n. 582, ago. 1976; Informe Económico y Financiero, primera quincena, marzo, 1987.

BARANDIARÁN, E. La crisis financiera chilena. Documenıo de Trabajo, n. 6, oct. 1988.

CAGAN, P. The monetary dynamics of hyper inflation. In: FRIEDMAN, M., ed. Studies in the Quarterly Theory of Money, Chicago, The University of Chicago Press, 1956.

CHOW, G. C. Econometrics. New York, McGraw-Hill, 1983.

COOLEY, T. T. \& PRESCOTT, E. C. Vaying parameters regression: a theory and some applications. Annals of Economic and Social Measurement. v. 2, n. 4, oct. 1973.

CORBO, V. Inflation in Developing Countries. Amsterdam, North-Holland, cap. 10: 179-96, 1974.

- Dos ensayos sobre demanda por dinero en Chile. Documento de Trabajo, 73. Pont. Universidad Católica de Chile, 1981.

\& McNELIS, P. D. The pricing of manufactured goods 
during trade liberalization: evidence from Chile, Israel and Korea. DRD Discussion Paper, World Bank, jun. 1987.

ENGLE, R. F \& WATSON, M. W. Applications of Kalman Filtering in econome trics, oct. 1985. Mimeo.

FAIR, R. C. Specification, Estimation and Analysis of Macroeconometrics Models. Cambridge, Harvard University Press, 1984.

HARVEY, A. C. Time Series Models. Phillip Allan, 1981.

\& COLLIER, P. Testing for functional misspecification in regression analysis. Journal of Econometrics, p. 103-19, 1977.

JOHNSTON, J. Econometrics Methods. McGraw-Hill, New York, 1985.

LABAN, R. La evolución de la demanda por dinero en Chile (1974. -86): una aplicación del Filtro de Kalman. Tesis de Magister en Ciencias Económicas, Pontifícia Universidad Católica de Chile, 1987a.

El teorema de extracción de señales y la estimación de parámetros variables: Una nota. Cuadernos de Economia, 73. Pont. Universidad Católica de Chile, $1987 \mathrm{~b}$.

LARRAIN, F. Anatomía de la crisis económica de Chile. Ambito Financiero. Buenos Aires, septiembre, 1985.

LUCAS, R. Econometric policy evaluation: a critique. In: BRUNNER, K. \& MELTZER, A., eds. The Phillips Curve and Labor Markets, Carnegie Rochester Series on Public Policy, p. 19-46, 1976.

MADDALA, G. S. Econometrics, McGraw-Hill, 1977.

MATTE, R. \& ROJAS. P. Evolución reciente del mercado monetario en Chile y una estimación de la demanda por dinero. Banco Central de Chile. Santiago, dic. 1986. Mimeo.

PHILLIPS, G. D. A. \& HARVEY, A. C. A simple test for serial correlation in regression analysis. Journal of the American Statistical Association, v. 69, 1974.

ROLEY, V. Money demand predictibility. Journal of Money, Credit and Banking, v. 17, n. 4, parte 2, 1985.

SARGENT, T. J. A classical macroeconometric model for the United States. In: LUCAS, R. \& SARGENT, T., eds. Rational Expectations and Econometric Practice. University of Minnesota, 1981.

SARRIS, S. A bayesian approach to estimation of time-varying regression coeficients. Annals of Economic and Social Measurement, v. 2, n. 4: 501-23, oct. 1973.

VIAL, J. \& MARIN, M. B. Series monetarias chilenas: 1960-1985. Estudio de Economía, v. 13, n. 1, Universidad de Chile, Santiago, abr. 1986.

WATSON, P. K. Kalman filtering as an alternative to ordinary least squares - some theoretical considerations an empirical results. Empirical Economics, 8, 1983. 